\title{
Adaptive-resolution reinforcement learning with polynomial exploration in deterministic domains
}

\author{
Andrey Bernstein • Nahum Shimkin
}

Received: 7 July 2009 / Revised: 25 March 2010 / Accepted: 16 April 2010 / Published online: 5 May 2010 (C) The Author(s) 2010

\begin{abstract}
We propose a model-based learning algorithm, the Adaptive-resolution Reinforcement Learning (ARL) algorithm, that aims to solve the online, continuous state space reinforcement learning problem in a deterministic domain. Our goal is to combine adaptiveresolution approximation schemes with efficient exploration in order to obtain polynomial learning rates. The proposed algorithm uses an adaptive approximation of the optimal value function using kernel-based averaging, going from coarse to fine kernel-based representation of the state space, which enables us to use finer resolution in the "important" areas of the state space, and coarser resolution elsewhere. We consider an online learning approach, in which we discover these important areas online, using an uncertainty intervals exploration technique. In addition, we introduce an incremental variant of the ARL (IARL), which is a more practical version of the original algorithm with reduced computational complexity at each stage. Polynomial learning rates in terms of mistake bound (in a PAC framework) are established for these algorithms, under appropriate continuity assumptions.
\end{abstract}

Keywords Reinforcement learning · Adaptive resolution · Kernel functions · Efficient exploration

\section{Introduction}

Markov Decision Processes (MDPs) provide a standard framework for handling sequential decision problems under uncertainty (Bertsekas 2007; Puterman 1994). Solid theory and a variety of algorithms enable the efficient computation of the optimal value function and optimal control policies in MDPs when the state and action spaces are finite. However, an exact solution becomes intractable when the number of states is large or infinite. In this case,

Editor: Roni Khardon.

A. Bernstein $(\bowtie) \cdot$ N. Shimkin

Department of Electrical Engineering, Technion-Israel Institute of Technology, Haifa, Israel

e-mail: andreyb@tx.technion.ac.il

N. Shimkin

e-mail: shimkin@ee.technion.ac.il 
some approximation schemes are required. See Boutilier et al. (1999), Bertsekas (2007), Powell (2007), for a general discussion and overview.

A well-established approximation approach is that of value function approximation using a finite set of basis functions, or kernels. A particular case is state aggregation, in which the state space is represented by a finite (and relatively small) collection of cells. Each cell is said to aggregate the states that fall in it. Once the aggregation is performed, the new problem is a planning problem in a reduced state space, which can be solved by regular techniques. The main question that arises here is how to perform the aggregation, so that, on the one hand, we obtain a "good" approximation of an optimal policy, and on the other hand reduce the problem complexity. This question was addressed in many papers, such as Whitt (1978), Chow and Tsitsiklis (1991), which provide formal answers under some continuity assumptions on the model parameters.

An extra difficulty is added when dealing with learning problems, namely situations where the MDP model is initially unknown. Reinforcement Learning (RL) encompasses a wide range of techniques for solving this problem by interacting with the environment. An important part of an RL algorithm is the exploration scheme. The role of exploration is to gain new information by appropriate action selection which directs the agent towards unknown states of the MDP.

For finite state and action spaces, efficient learning algorithms were presented and proved to learn nearly optimal behavior (with high probability) within a time or error bound that is polynomial in the problem size. These include the $E^{3}$ (Kearns and Singh 2002), R-MAX (Brafman and Tennenholtz 2002), MBIE (Strehl and Littman 2005), UCRL (Auer and Ortner 2006), GCB (Chapman 2007) and OLP (Tewari and Bartlett 2007) algorithms. These algorithms use efficient exploration techniques, which are often based on the so-called "optimism in face of uncertainty" principle. However, these algorithms are infeasible in cases where the state and/or action spaces are very large or infinite, since their time and space complexity is typically polynomial in the size of the space.

Several new contributions to the problem of RL in continuous state space which are related to adaptive aggregation and kernel-based methods appeared in the literature. The proposed algorithms can be roughly divided into two groups. The first group contains algorithms that are heuristic in nature. Moore and Atkeson (1995) proposed a learning algorithm for deterministic domains, which uses adaptive aggregation of the state space. Munos and Moore (2002) discussed the use of adaptive aggregation for the planning problem in deterministic domains, with particular focus on the introduction and evaluation of a wide variety of possible splitting criteria. Jong and Stone (2006) combined an exploration technique with kernel-based function approximation. Loth et al. (2006) use optimistic policy iteration with some kernelized gradient-type algorithm. Bonarini et al. (2005) introduced a learning algorithm which builds a multi-resolution state representation by using sophisticated splitting and merging rules, and showed experimentally how this algorithm can scale to large stochastic domains. Recently, a multi-resolution exploration algorithm was proposed by Nouri and Littman (2008). The algorithm uses state aggregation and employs a splitting mechanism that is based on the number of visits to a particular state-action cell and on its size. We note that in these papers, no convergence and learning rate guarantees were provided.

The algorithms in the second group provide some convergence results without treating explicitly the exploration-exploitation problem. Ormoneit and Sen (2002) introduced an approximate kernel-based Bellman operator and showed that theoretical guarantees can be obtained when a set of independent transition samples is used in order to define this operator. Konda and Tsitsiklis (2003) proposed and analyzed a class of actor-critic algorithms, using 
a parameterized set of randomized stationary policies. In order to avoid the explorationexploitation tradeoff, it is assumed that under every policy there is a positive probability to choose any action at every state. Munos and Szepesvári (2008) introduced a variant of the fitted value iteration method (using a finitely parameterized class of bounded functions), which uses a generative model (or simulator) of the environment in order to obtain samples. Antos et al. (2008) proposed an instance of the fitted policy iteration method, which employs a modified form of Bellman residual minimization procedure to approximate the action-value function. The algorithm learns from a single trajectory of some given fixed policy. We note that these algorithms are offline in the sense that they learn from a given trajectory, and hence do not deal with the exploration-exploitation trade-off which is central in the present paper.

An important class of control and learning problems deals with deterministic models over a continuous (infinite) state space. The examples of such problems and applications of RL algorithms to them are the "Car On Hill" problem (see for instance Sutton 1996; Moore and Atkeson 1995), the inverted pendulum and cart-pole problems (as in Doya 2000; Sutton 1996), and the "Acrobat" problem (Sutton 1996). The applied RL algorithms were heuristic extensions of the classical finite state space algorithms using various kinds of approximation schemes.

In this paper we consider the online reinforcement learning problem in MDPs with large or infinite state space, finite action space, discounted return criterion, and with deterministic dynamics and rewards. Our goal is to combine adaptive-resolution approximation schemes with efficient exploration in order to obtain fast (polynomial) learning rates. For concreteness we will focus on the continuous state case; however our schemes and results also apply to the discrete case, where the number of states is very large or countably infinite. The proposed algorithms use an adaptive approximation of the optimal value function using kernelbased averaging, going from coarse to fine kernel-based representation of the state space, which enables us to use finer resolution in the "important" areas of the state space, and coarser resolution elsewhere. We consider an online learning approach, in which we discover these important areas online, using an uncertainty intervals exploration technique. ${ }^{1}$ Certain continuity assumptions on the basic model parameters will be imposed. Such assumptions are essential for generalization in continuous state space. We note that we focused here on deterministic domains for simplicity, and in order to emphasize the aspects related to adaptive resolution. However, we believe that the proposed approach and results can be extended to stochastic systems by employing stochastic confidence intervals in addition to deterministic uncertainty intervals.

There is a wide use of the term "kernel" in the literature. Our usage of kernels mostly resembles that of Ormoneit and Sen (2002) in the sense that the kernels are used as local basis functions; however, for computational purposes, we focus on finite support kernels rather than on Gaussian-type ones. From this perspective, our kernel-based approach can be compared with spline-based approximations of signals, which are recently widely used in signal and image processing field (Unser 1999). We also note that a similar use of kernel functions in the case of a finite state space was proposed by Singh et al. (1995), in the context of soft state aggregation.

The principle that governs our scheme is simply to split frequently visited kernels. The idea behind this principle is as follows. As time progresses, we will visit states in the support

\footnotetext{
${ }^{1}$ Our uncertainty intervals are the analogue of the confidence intervals used in the stochastic case (Strehl and Littman 2005). However, the origin of uncertainty in our model is the (deterministic) aggregation error, rather than stochastic sampling error.
} 
of kernels that are "close" to the optimal trajectory; and on the optimal trajectory, we need high resolution.

Our focus in this paper is on the combination of the adaptive resolution scheme with efficient exploration that leads to fast (polynomial) learning rates. As a metric for the learning rates in our algorithms we will use the total mistake count. This metric counts the total number of time-steps in which the algorithm's computed policy is strictly suboptimal for the current state. This metric has been used in a number of recent works on online learning in discounted MDP problems ${ }^{2}$ (Kakade 2003; Strehl and Littman 2005; Strehl et al. 2006b). In our case we will establish two types of mistake bounds, which we call the prior bound and the posterior bound. The first type ensures that our algorithm is not worse than a non-adaptive algorithm, which uses a single set of uniformly dense kernels. In this case, our mistake bound is polynomial in the number of kernels in the latter set. The second type ensures that the total mistake count is polynomial in the number of actually used kernels (throughout the algorithm operation). We note that, to our best knowledge, these are the first online performance bound results for kernel-based RL algorithms in continuous state spaces, for both non-adaptive and adaptive case.

The algorithm proposed in this paper is related to the Adaptive Aggregation Algorithm (AAA) introduced in Bernstein and Shimkin (2008) (see also Bernstein 2007). In the AAA, constant kernels are used, leading to adaptive state aggregation. Therefore, the value function approximation scheme proposed in this paper is a generalization of that of the AAA. However, the proposed algorithm itself is different since we have eliminated the need of the so-called "virtually visited cells" by using an improved dynamic programming operator. Hence, the obtained algorithm is simpler than AAA (both conceptually and computationally).

The paper is structured as follows. In Sect. 2 we present the model and notation. In Sect. 3 we introduce some further definitions and assumptions. In Sect. 4 we propose our general algorithm, while Sect. 5 discusses some computational aspects of the proposed algorithm. In Sect. 6 polynomial bounds on the total mistake count of the algorithm are presented, while Sect. 7 proves these bounds. Section 8 introduces an incremental variant of our algorithm, which performs only a few back-ups of value iteration at each time-step, instead of performing exact calculation. Its analysis is presented in Sect. 9. Section 10 discusses the applicability of the algorithm and its assumptions to a typical deterministic control problem, namely to the problem of inverted pendulum, and proposes some improvements and extensions that can increase the practical efficiency of the algorithm. Finally, conclusions and future work are presented in Sect. 11.

\section{Model and performance metric}

We denote a deterministic MDP by the 4-tuple $M=(\mathbb{X}, \mathbb{A}, f, r)$, where $\mathbb{X}$ is a state space, $\mathbb{A}$ is an action space, $f(x, a)$ is the transition function which specifies the next state $x^{\prime} \in \mathbb{X}$ given the previous state $x \in \mathbb{X}$ and action $a \in \mathbb{A}$, and $r(x, a)$ is the immediate reward function which specifies the reward of performing action $a \in \mathbb{A}$ in state $x \in \mathbb{X}$. We assume that $r(x, a) \in\left[r_{\text {min }}, r_{\text {max }}\right]$, a finite and known interval.

Let $d: \mathbb{X} \times \mathbb{X} \rightarrow \mathbb{R}$ be a fixed metric on $\mathbb{X}$. We assume the following regarding the state and action spaces.

\footnotetext{
${ }^{2}$ These works refer to this metric as the sample complexity of exploration.
} 


\section{Assumption 1}

1. The action space $\mathbb{A}$ is a finite set.

2. The state space $\mathbb{X}$ is a bounded subset of $\mathbb{R}^{n}$. Thus, there exists a constant $\Delta_{\max }<\infty$ such that for all $x, x^{\prime} \in \mathbb{X}, d\left(x, x^{\prime}\right) \leq \Delta_{\max }$.

The MDP $M$ is used to model a dynamic environment, or a dynamic system, with which a learning agent interacts. The interaction proceeds as follows: At time $t$ the agent observes the state $x_{t} \in \mathbb{X}$, chooses an action $a_{t} \in \mathbb{A}$, receives a reward $r_{t}=r\left(x_{t}, a_{t}\right)$, and the process moves to state $x_{t+1}=f\left(x_{t}, a_{t}\right)$.

Let $h_{t} \triangleq\left\{x_{0}, a_{0}, x_{1}, a_{1}, \ldots, x_{t-1}, a_{t-1}, x_{t}\right\}$ denote the history of observed states and actions, that is available to the agent at time $t$ to make its choice of $a_{t}$. Also, let $\mathbb{H}_{t} \triangleq$ $(\mathbb{X} \times \mathbb{A})^{t} \times \mathbb{X}$ denote the space of all possible histories up to time $t$. Then, at each time $t$, the agent makes its decision according to some decision rule $\pi_{t}: \mathbb{H}_{t} \rightarrow \mathbb{A}$, so that $a_{t}=\pi_{t}\left(h_{t}\right)$, $t \geq 0$. The collection $\pi=\left\{\pi_{t}\right\}_{t=0}^{\infty}$ is the control policy. A policy is stationary if the decision rule does not change over time, and depends only on the last state observed. We shall slightly abuse notation and identify the stationary policy $\pi$ with the map $\pi: \mathbb{X} \rightarrow \mathbb{A}$, so that at each time $t, a_{t}=\pi\left(x_{t}\right)$.

In this paper we focus on the discounted return criterion. For a given initial state $x_{0}=x$, we denote the infinite horizon discounted return of state $x$, for a given policy $\pi$, in MDP $M$, by

$$
J_{M}^{\pi}(x) \triangleq \sum_{t=0}^{\infty} \gamma^{t} r\left(x_{t}, \pi_{t}\left(h_{t}\right)\right),
$$

where $0<\gamma<1$ is the discount factor. The optimal return is denoted by $V_{M}(x) \triangleq$ $\sup _{\pi} J_{M}^{\pi}(x)$, which is also called the optimal value function. We often drop $M$ from the notation above if it does not cause confusion. A policy $\pi$ is optimal if $J^{\pi}(x)=V(x)$ holds for all $x \in \mathbb{X}$. For any $\epsilon>0$, a policy $\pi$ is $\epsilon$-optimal if $J^{\pi}(x) \geq V(x)-\epsilon$ holds for all $x \in \mathbb{X}$.

In what follows, we let $V_{\max } \triangleq \frac{r_{\max }}{1-\gamma}$. Note that $V_{\max }$ is the maximal possible discounted return of any policy. Also, let

$$
V_{b} \triangleq \frac{1}{1-\gamma}\left(r_{\max }-r_{\min }\right)
$$

denote the maximal difference between the returns of any two policies.

It is well known (Puterman 1994) that the optimal value function satisfies Bellman's equation

$$
V(x)=\max _{a \in \mathbb{A}}\{r(x, a)+\gamma V(f(x, a))\}, \quad x \in \mathbb{X},
$$

and that any stationary deterministic policy $\pi^{*}$ which satisfies

$$
\pi^{*}(x) \in \operatorname{argmax}_{a \in \mathbb{A}}\{r(x, a)+\gamma V(f(x, a))\}, \quad x \in \mathbb{X},
$$

is an optimal policy. Let $Q(x, a) \triangleq r(x, a)+\gamma V(f(x, a))$ denote the action-value function, or $Q$-function, which provides the return of choosing an action $a$ in state $x$, and then following an optimal policy. 
To assess the learning rate of our algorithms, two performance metrics will be considered. The first and simpler one is the total (action) mistake count, which is given by

$$
\left.\operatorname{AMC}(\epsilon) \triangleq \sum_{t=0}^{\infty} \mathbb{I}\left\{Q\left(x_{t}, a_{t}\right)<V\left(x_{t}\right)-\epsilon\right)\right\}, \quad \epsilon \geq 0 .
$$

This metric counts the number of sub-optimal actions, that is, the number of times that an algorithm executed an action whose action-value is $\epsilon$-inferior to the optimal value. Observe that for $\epsilon=0, \mathrm{AMC}(0)$ is simply the total number of non-optimal actions taken in the course of learning. For deterministic domains with finite state-space, we then have the following near-optimality criterion.

Definition 1 (Action Mistake Bound) Assume that $|\mathbb{X}|$ is finite. A learning algorithm is $P A C$ (Probably Approximately Correct) $^{3}$ if there exists a polynomial

$$
B=B\left(|\mathbb{X}|,|\mathbb{A}|, \frac{1}{1-\gamma}, \frac{1}{\epsilon}\right)
$$

such that for all $\epsilon>0, \operatorname{AMC}(\epsilon)<B$.

In the above definition, the bound $B$ depends on the number of states $|\mathbb{X}|$. In case $|\mathbb{X}|$ is infinite, some other measures of $\mathbb{X}$ must be considered. As already mentioned, in our case we will replace $|\mathbb{X}|$ by the cardinality of the set of sufficiently dense kernels over the state space.

A second metric of interest is the policy-mistake count, which counts the number of time steps $t$ in which the algorithm executes a non $\epsilon$-optimal policy. Specifically, let $\pi_{t}: \mathbb{H}_{t} \rightarrow \mathbb{A}$ be the decision rule that the algorithm uses at time $t$ to choose its action. Then, given $h_{t}, \mathcal{A}_{t}=\left\{\pi_{k}\right\}_{k=t}^{\infty}$ is a (non-stationary) policy that the algorithm implements at time $t$, and $\sum_{k=t}^{\infty} \gamma^{k-t} r_{k} \triangleq J^{\mathcal{A}_{t}}\left(x_{t}\right)$ can be interpreted as the return of this policy from time $t$ onward, where $r_{k}=r\left(x_{k}, \pi_{k}\left(x_{k}\right)\right)$ and $x_{k+1}=f\left(x_{k}, \pi_{k}\left(x_{k}\right)\right)$. Now, the policy-mistake count is defined as

$$
\operatorname{PMC}(\epsilon) \triangleq \sum_{t=0}^{\infty} \mathbb{I}\left\{J^{\mathcal{A}_{t}}\left(x_{t}\right)<V\left(x_{t}\right)-\epsilon\right\} .
$$

It is easily verified (see Corollary 1 in Bernstein 2007) that policy-mistake count is a stronger criterion than action-mistake count, in the sense that $\operatorname{AMC}(\epsilon) \leq \operatorname{PMC}(\epsilon)$. Hence we will state all bounds below in terms of the PMC, with the understanding that they apply to the AMC as well.

\section{Preliminaries}

In this section, we formally define kernel functions and splitting schemes that are employed in our algorithms, and introduce a continuity assumption on the basic model parameters.

\footnotetext{
${ }^{3}$ Note, that while the "probably" aspect is absent in our deterministic case, we will find it convenient to keep the PAC terminology. 


\subsection{Kernel functions}

Our algorithm uses an approximation of the optimal value function $V(x)$ using kernel-based averaging. As was already mentioned, we use the term "kernel" to denote any local basis function $\phi: \mathbb{R}^{n} \rightarrow \mathbb{R}^{+}$. We shall focus in this work on finite support kernels. Recall that the support of a function $\phi: \mathbb{R}^{n} \rightarrow \mathbb{R}^{+}$is defined as $\operatorname{supp}(\phi) \triangleq c \ell\left\{x \in \mathbb{R}^{n}: \phi(x) \neq 0\right\}$, where $c \ell\{\cdot\}$ denotes the closure of the set. Let

$$
\Delta(\phi) \triangleq \sup _{x, x^{\prime} \in \operatorname{supp}(\phi)} d\left(x, x^{\prime}\right)
$$

denote the diameter of the support of $\phi$ under metric $d$.

Now, our basic definition is the following.

Definition 2 (Feasible Kernel Set) A set $\Phi=\left\{\phi_{i}\right\}_{i=1}^{m}$ of functions $\phi_{i}: \mathbb{R}^{n} \rightarrow \mathbb{R}^{+}$is called a feasible kernel set for $\mathbb{X}$ if the following holds:

1. Finite support: $\Delta\left(\phi_{i}\right)<\infty$ for all $i=1, \ldots, m$, and

2. Uniform coverage: $\sum_{i=1}^{m} \phi_{i}(x)=1$, for all $x \in \mathbb{X}$.

This definition encompasses several standard types of kernels, such as strict state aggregation, soft state aggregation, splines, etc. We will discuss this in more detail below.

\subsection{Splitting schemes}

As we are interested in adaptive kernel-based approximation of the optimal value function, we specify the way we perform refinement of a given kernel.

Definition 3 (Uniform Splitting Scheme) A splitting (or refinement) scheme tells us how to split any given kernel function $\phi$ into $K \geq 2$ new kernel functions $\phi_{1}, \ldots, \phi_{K}$. We say that the splitting scheme is uniform if there exists $0<\lambda<1$ such that for any given kernel function $\phi$ it holds that

$$
\Delta\left(\phi_{j}\right) \leq \lambda \Delta(\phi), \quad j=1, \ldots, K,
$$

and

$$
\sum_{j=1}^{K} \phi_{j}(x)=\phi(x) .
$$

Now, given a fixed uniform splitting scheme and an initial kernel set $\Phi_{0}$ for $\mathbb{X}$, we define the achievable class of kernel sets as the set of all kernel sets that can be obtained by repeatedly using the given splitting scheme starting from $\Phi_{0}$. Observe that all such kernel sets are feasible by the virtue of Definition 3. We note that both the initial kernel set $\Phi_{0}$ and the splitting scheme will be fixed in the course of the algorithm.

Several standard types of kernels fit the above definitions. We list below two of such types.

Splines In spline-based approximations of signals, a one-dimensional kernel (or spline) is a scaling and a dilation of some basic finite support function $\varphi^{(k)}: \mathbb{R}^{+} \rightarrow \mathbb{R}^{+}$, that is

$$
\phi(x)=A \varphi^{(k)}\left(\frac{x}{b}\right) \quad \text { for some } b>0 \text { and } A>0 .
$$


Fig. 1 Triangular kernels. Dotted line is the original kernel of support $\Delta$. Solid line is the resulting kernels after the split

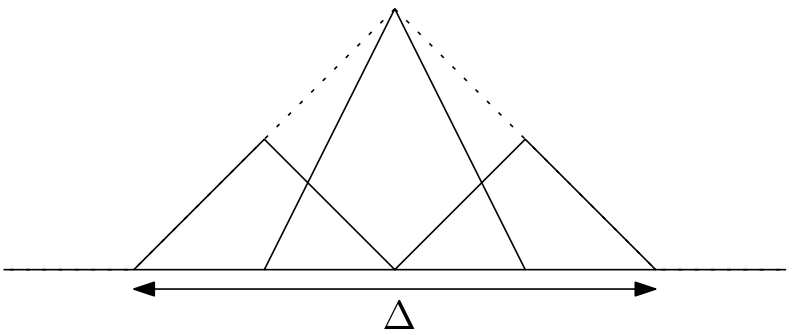

Here, $\varphi^{(k)}$ is called a basic spline (or, B-spline) of degree $k$ and is formed by the $(k+1)$ fold convolution of a rectangular pulse $\varphi^{(0)}(x) \triangleq \mathbb{I}\{|x| \leq 0.5\}$. In particular, $\varphi^{(0)}$ leads to a piecewise-constant approximation (i.e. aggregation), $\varphi^{(1)}$ - to a piecewise-linear approximation, and so on.

One of the advantages of splines is that there exist computationally efficient splitting schemes for them, which fit Definition 3. For example, consider the B-spline of degree 1 shown in Fig. 1(a). A standard B-spline "pyramid" (in the terminology of Unser 1999) is shown in Fig. 1(b). As can be observed, the original spline of support $\Delta$ was split to three splines of support $\Delta / 2$, in order to satisfy the condition that the sum of the new splines equals to the original one. See Unser (1999) for further details.

Normalized kernel functions A different approach is to use (a-priori) normalized kernel functions, as for example in Ormoneit and Sen (2002). That is,

$$
\phi_{i}(x)=\frac{\varphi_{i}\left(d\left(x, x_{i}\right)\right)}{\sum_{j=1}^{m} \varphi_{j}\left(d\left(x, x_{j}\right)\right)}, \quad i=1, \ldots, m,
$$

where $\varphi_{i}: \mathbb{R}^{+} \rightarrow \mathbb{R}^{+}$are some basic function with finite support, and $\left\{x_{i}\right\}_{i=1}^{m}$ are the points at which the kernels are centered (or, "placed"). We note that the well-known Cerebellar Model Articulation Controller (CMAC) (Albus 1975) fits into above framework. The advantage of these kernel functions is in their inherent normalization property. However, the splitting schemes for such kernels are not trivial in general, and will not be discussed here.

To summarize, any kernels that satisfy Definitions 2 and 3 can be used. However, in what follows, we will mostly focus on the spline-type kernels due to the well-established splitting schemes associated with them.

\subsection{Continuity assumption}

The following continuity property will be assumed to hold for the basic model parameters.

Assumption 2 There exist constants $\alpha>0$ and $\beta>0$ such that, for all $x_{1}, x_{2} \in \mathbb{X}$ and $a \in \mathbb{A}$,

$$
\begin{aligned}
& \left|r\left(x_{1}, a\right)-r\left(x_{2}, a\right)\right| \leq \alpha \cdot d\left(x_{1}, x_{2}\right), \\
& d\left(f\left(x_{1}, a\right)-f\left(x_{2}, a\right)\right) \leq \beta \cdot d\left(x_{1}, x_{2}\right) .
\end{aligned}
$$

A continuity assumption of some kind is obviously essential for generalization in continuous state spaces. Assumptions of similar nature to the one above were used in various works on state aggregation, such as Whitt (1978), Chow and Tsitsiklis (1991). However, we 
note that the specific assumptions used in these papers refer to continuity of probability densities. Consequently they are too strong for the continuous deterministic case as they imply that all states are mapped to the same target state.

We assume that both $\alpha$ and $\beta$ are known and given to the learning algorithm.

Continuity of the basic model parameters implies continuity of the optimal value function. Specifically, we define the modulus of continuity of the optimal value function, and a modulus of continuity bound as follows.

Definition 4 The modulus of continuity of the optimal value function $V$ is defined as

$$
\omega(z) \triangleq \sup _{x_{1}, x_{2}: d\left(x_{1}, x_{2}\right) \leq z}\left|V\left(x_{1}\right)-V\left(x_{2}\right)\right|, \quad z>0 .
$$

Definition 5 Modulus of continuity bound (MCB) is a function $\bar{\omega}(z)$ which satisfies:

1. $\omega(z) \leq \bar{\omega}(z), \forall z>0$,

2. $\bar{\omega}(z) \rightarrow 0$, as $z \rightarrow 0$.

In particular, the following lemma specifies an MCB in case $\gamma \beta \neq 1$. Its proof is presented in Sect. 7.1.

Lemma 1 For any given $x_{1}, x_{2} \in \mathbb{X}$, we have that

$$
\left|V\left(x_{1}\right)-V\left(x_{2}\right)\right| \leq \bar{\omega}\left(d\left(x_{1}, x_{2}\right)\right),
$$

where $\bar{\omega}$ is defined as follows:

1. If $\gamma \beta<1$ (non-expansive case),

$$
\bar{\omega}(z)=\frac{\alpha}{1-\gamma \beta} z
$$

2. If $\gamma \beta>1$ (expansive case),

$$
\bar{\omega}(z)=c z^{\log _{\beta}(1 / \gamma)},
$$

where

$$
c \triangleq 2 \beta\left(\frac{\alpha}{\gamma \beta-1}\right)^{\log _{\beta}(1 / \gamma)} V_{b}^{\log _{\beta}(\gamma \beta)} .
$$

Here $\alpha, \beta$ are taken from Assumption 2, $\gamma$ is the discount factor, and $V_{b}$ is defined in (1).

Remark Note that there is a substantial difference in the nature of bounds (7) and (8). This difference can be understood by observing the bound on the distance between optimal values of two states (see the proof of Lemma 1 in Sect. 7 for details):

$$
\left|V\left(x_{1}\right)-V\left(x_{2}\right)\right| \leq \alpha d\left(x_{1}, x_{2}\right) \sum_{k=0}^{H-1}(\gamma \beta)^{k}+\gamma^{H} V_{b}, \quad H>0 .
$$

If $\gamma \beta>1$, instead of bounding the infinite sum of distances between future rewards, we have to employ a "cut-off tactics". Specifically, we have to make a balance between the first term in (10), which grows exponentially in $H$, and the second term, which decays exponentially in $H$. This results in a more complex bound $\bar{\omega}(z)$, with a worse dependence on $z$.

We assume that a fixed bound $\bar{\omega}$ that satisfies Definition 5 is used throughout. 


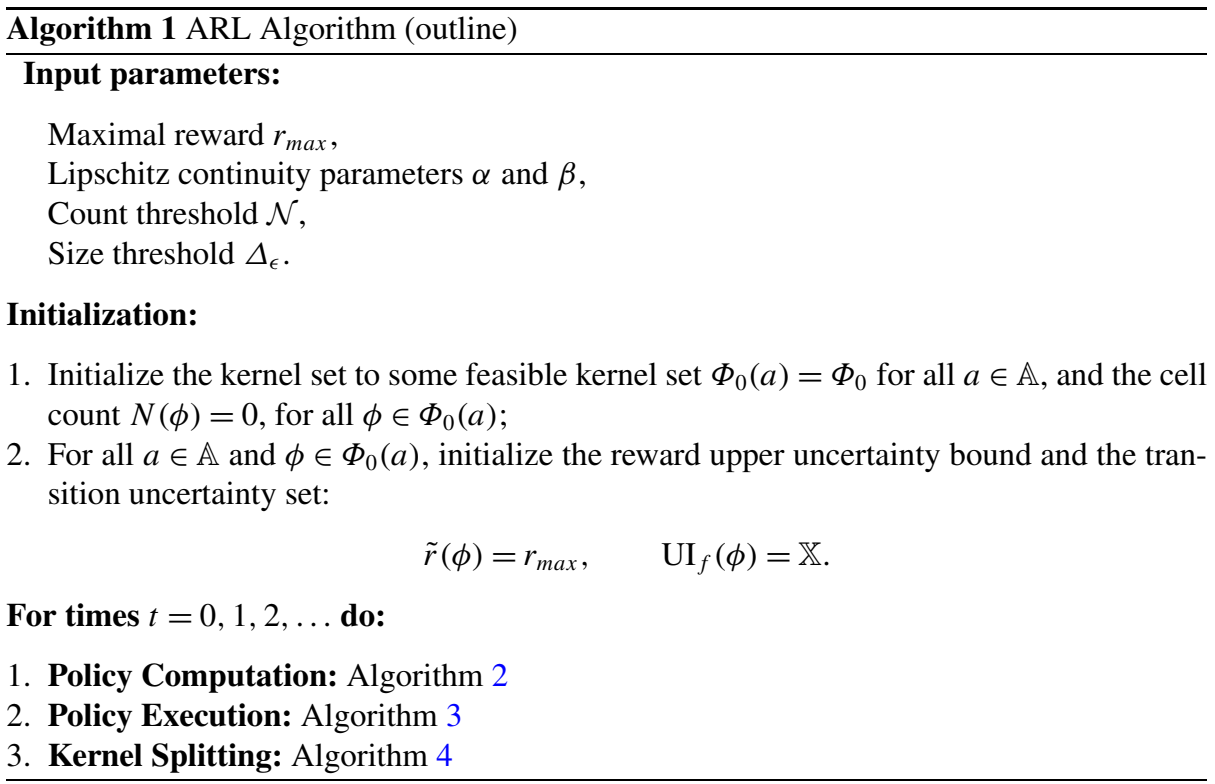

\section{The ARL algorithm}

In this section we present the Adaptive-resolution Reinforcement Learning (ARL) algorithm, which is directly based on the principle of refinement of frequently visited areas of the state-space. In the following subsections we present the different components of this algorithm in detail. An outline of the complete algorithm is presented as Algorithm 1.

\subsection{Action kernel sets}

Our algorithm will employ a separate kernel set for every action. This will allow the use of different resolutions for the different actions. We denote by

$$
\Phi_{t}(a) \triangleq\left\{\phi_{i}^{a}(x)\right\}_{i=1}^{m_{a}}
$$

the kernel set that is used by the algorithm at time $t$ for action $a$, with $m_{a} \triangleq\left|\Phi_{t}(a)\right|$. Also, for each $a \in \mathbb{A}$ and $\phi \in \Phi_{t}(a)$, we let

$$
N_{t}(\phi) \triangleq \sum_{\tau=0}^{t-1} \mathbb{I}\left\{\left(x_{\tau}, a_{\tau}\right)=(x, a): x \in \operatorname{supp}(\phi)\right\}
$$

denote the number of times (up to time $t$ ) the process visited a state in the support of $\phi$ and the action $a$ was taken. In our presentation we will frequently refer to this latter event using the terminology "a kernel $\phi \in \Phi_{t}(a)$ is visited" or "a kernel $\phi \in \Phi_{t}(a)$ was encountered".

In what follows, we often drop the time index from our notation for ease of exposition.

\subsection{Empirical model}

We use a single sample to estimate empirically the reward and transition at the support of each kernel. Specifically, suppose that we choose action $a$ in state $x$. We thus obtain the 
sample $\left(x, a, r=r(x, a), x^{\prime}=f(x, a)\right)$. Now, for each $\phi \in \Phi_{t}(a)$ having $x \in \operatorname{supp}(\phi)$, we define the empirical model based on this single sample:

$$
\begin{gathered}
\hat{r}(\phi)=r, \\
\hat{f}(\phi)=x^{\prime} .
\end{gathered}
$$

There are several options how to maintain the model in the course of learning:

1. Once the sample for $\phi$ is obtained, the model remains unchanged for this kernel (until the kernel is split).

2. A more effective strategy is to replace the model of a kernel $\phi$ using a better sample (according to $\phi$ ). For instance, if the current model is based on the sample

$$
\left(x_{1}, a, r_{1}=r\left(x_{1}, a\right), x_{1}^{\prime}=f\left(x_{1}, a\right)\right),
$$

and a new sample

$$
\left(x_{2}, a, r_{2}=r\left(x_{2}, a\right), x_{2}^{\prime}=f\left(x_{2}, a\right)\right)
$$

arrives, we will update the model using the new sample if $\phi\left(x_{2}\right)>\phi\left(x_{1}\right)$ (assuming $\phi$ is larger in the central part of its support, as is the typical case).

3. Moreover, we can save all the samples obtained for $\phi$ (even if we do not use them for the model). When $\phi$ will be split, these samples should allow us to initialize the model of the new kernels.

We note that the algorithm and its analysis remain unchanged, regardless of the choice above. For ease of exposition, we assume throughout that the first option is used.

\subsection{Confidence intervals and upper value function}

In our algorithm we will use an uncertainty intervals exploration technique as it applies to deterministic systems due to finite approximation.

Recall that $\Delta(\phi)$ denotes the diameter of the support of a kernel function $\phi(5)$. At any time $t$, and for every $a \in \mathbb{A}$ and $\phi \in \Phi_{t}(a)$, we define the reward uncertainty interval around the empirical reward (11) as:

$$
\mathrm{UI}_{r}(\phi) \triangleq[\hat{r}(\phi)-\alpha \Delta(\phi), \hat{r}(\phi)+\alpha \Delta(\phi)]
$$

if the pair $(\phi, a)$ was sampled till time $t$; otherwise, the reward uncertainty interval for this kernel is inherited from the parent kernel. By the continuity Assumption 2, this uncertainty interval satisfies that $r(x, a) \in \mathrm{UI}_{r}(\phi)$ for all $x \in \operatorname{supp}(\phi)$. In our algorithm only the upper bound of $\mathrm{UI}_{r}(\phi)$ will be used, which we denote by

$$
\tilde{r}(\phi) \triangleq \hat{r}(\phi)+\alpha \Delta(\phi)
$$

Next, the transition uncertainty set is defined as:

$$
\mathrm{UI}_{f}(\phi) \triangleq\{y \in \mathbb{X}: d(y, \hat{f}(\phi)) \leq \beta \Delta(\phi)\}
$$

If the pair $(\phi, a)$ was not sampled till time $t$, the uncertainty set is inherited from the parent kernel as in the reward case. Again, by the continuity assumption, this uncertainty set 
Fig. 2 An example of transition uncertainty set in a special case of kernels with disjoint supports (that is, strict state aggregation). This set is constructed based on the sample $\left(x, x^{\prime}=\hat{f}(\phi)\right)$ with $x \in \operatorname{supp}(\phi)$. The shaded area inside the circle is the uncertainty set $\mathrm{UI}_{f}(\phi)$

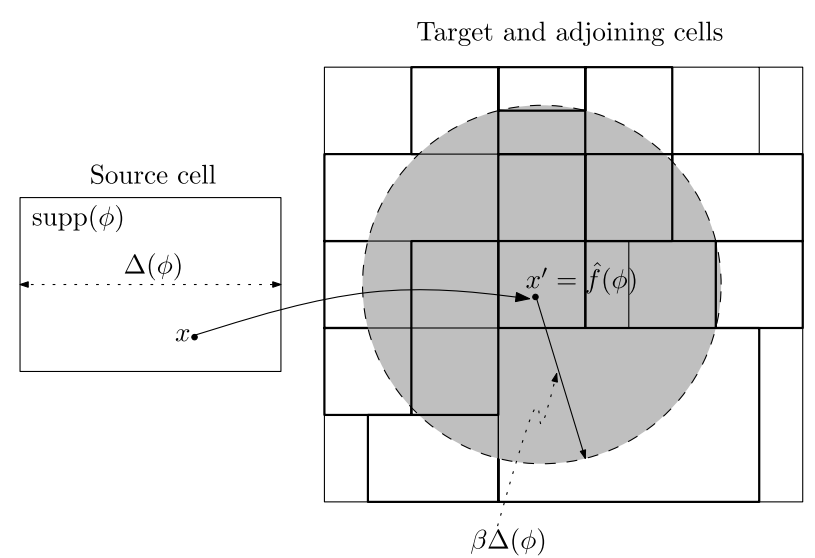

satisfies: $f(x, a) \in \mathrm{UI}_{f}(\phi)$ for all $x \in \operatorname{supp}(\phi)$. The concept of transition uncertainty set is shown in Fig. 2. Observe that for every $y, y^{\prime} \in \mathrm{UI}_{f}(\phi)$ we have that

$$
d\left(y, y^{\prime}\right) \leq d(y, \hat{f}(\phi))+d\left(y^{\prime}, \hat{f}(\phi)\right) \leq 2 \beta \Delta(\phi) .
$$

Using this notation, we define the following dynamic programming (DP) operator.

Definition 6 The upper DP operator at time $t$ is defined for any given function $g: \mathbb{X} \rightarrow \mathbb{R}$ by

$$
\mathcal{T} g(x)=\max _{a \in \mathbb{A}}\left\{\sum_{\phi \in \Phi_{t}(a)} \phi(x)\left(\tilde{r}(\phi)+\gamma\left[\min _{y \in \mathrm{UI}_{f}(\phi)} g(y)+\bar{\omega}(2 \beta \Delta(\phi))\right]\right)\right\},
$$

where $\bar{\omega}$ is an MCB (see Definition 5 and Lemma 1 ).

Now, using this operator, we define the upper value function (UVF) as the solution of the following fixed point equation:

$$
\widetilde{V}_{t}(x)=\mathcal{T} \widetilde{V}_{t}(x), \quad x \in \mathbb{X}
$$

We can show the following properties of (13) and its solution (see Sect. 7.2 for proofs).

Lemma 2 The operator $\mathcal{T}$ is a contraction mapping in the $\ell_{\infty}$ norm, with the contraction factor $\gamma$. Thus, there exists a unique solution to (13).

Lemma 3 The UVF $\widetilde{V}_{t}$ is indeed an upper bound on the optimal value function. That is, at every time $t$, we have that

$$
\widetilde{V}_{t}(x) \geq V(x), \quad \forall x \in \mathbb{X}
$$

We note that the unique solution of (13) is defined on an infinite state space $\mathbb{X}$, and its computation is not a trivial issue in general. We will discuss the computational aspects in Sect. 5. Also, observe that the operator $\mathcal{T}$ can be interpreted as an upper-bound approximation of the optimal Bellman's operator (cf. (2)) using a basis of kernel functions. Indeed, for 
$\phi \in \Phi_{t}(a)$, the term

$$
\widetilde{Q}_{t}(\phi) \triangleq \tilde{r}(\phi)+\gamma\left[\min _{y \in \mathrm{UI}_{f}(\phi)} \widetilde{V}_{t}(y)+\bar{\omega}(2 \beta \Delta(\phi))\right]
$$

is an upper-bound approximation of the optimal $Q$-function $Q(x, a)$ for all $x$ in the support of $\phi$. To see this, first note that $\tilde{r}(\phi)$ is an upper bound on the immediate reward that can be obtained for such $x$ using $a$. For the second term, observe that for every $y, y^{\prime} \in \mathrm{UI}_{f}(\phi)$ we have $d\left(y, y^{\prime}\right) \leq 2 \beta \Delta(\phi)$, which implies by the continuity of the optimal value function that

$$
V\left(y^{\prime}\right)-V(y) \leq \bar{\omega}(2 \beta \Delta(\phi)) .
$$

Now, since for every $x \in \operatorname{supp}(\phi), f(x, a) \in \mathrm{UI}_{f}(\phi)$, we have for such $x$ and all $y \in \mathrm{UI}_{f}(\phi)$ that

$$
V(f(x, a)) \leq V(y)+\bar{\omega}(2 \beta \Delta(\phi)) \leq \widetilde{V}_{t}(y)+\bar{\omega}(2 \beta \Delta(\phi))
$$

where the last inequality holds by Lemma 3 . Thus, $\min _{y \in \mathrm{UI}_{f}(\phi)} \widetilde{V}_{t}(y)+\bar{\omega}(2 \beta \Delta(\phi))$ is the tightest possible upper bound on the next state value among those in the transition uncertainty set of $\phi$.

In addition to providing an upper bound for $V$, we will show that the UVF converges to $V$ as the kernel supports shrink (Lemma 6 in Sect. 7.3).

The policy that is used in the algorithm is now the optimal (or greedy) policy with respect to $\widetilde{V}_{t}(x)$ :

$$
\pi_{t}(x)=\underset{a \in \mathbb{A}}{\operatorname{argmax}}\left\{\sum_{\phi \in \Phi_{t}(a)} \phi(x) \widetilde{Q}_{t}(\phi)\right\}, \quad x \in \mathbb{X} .
$$

We summarize the UVF and policy computation algorithm in Algorithm 2, and the policy execution process in Algorithm 3.

\section{Algorithm 2 Policy Computation}

If the model has changed (that is, some kernel has been visited for the first time, or some kernel has been split):

1. Compute the upper value function (UVF) by solving

$$
\widetilde{V}_{t}(x)=\mathcal{T} \widetilde{V}_{t}(x), \quad x \in \mathbb{X},
$$

where $\mathcal{T}$ is specified in Definition 6.

2. Compute the corresponding optimal policy

$$
\pi_{t}(x)=\underset{a \in \mathbb{A}}{\operatorname{argmax}}\left\{\sum_{\phi \in \Phi_{t}(a)} \phi(x) \widetilde{Q}(\phi)\right\}, \quad x \in \mathbb{X},
$$

where $\widetilde{Q}$ is defined in (14). If more than one action achieves the maximum, choose the first one in lexicographic order.

Otherwise, use the previously computed value and policy: $\widetilde{V}_{t}=\widetilde{V}_{t-1}$ and $\pi_{t}=\pi_{t-1}$. 
Algorithm 3 Policy Execution

Execute the action $a_{t}=\pi_{t}\left(x_{t}\right)$, and observe $r_{t}=r\left(x_{t}, a_{t}\right), x_{t+1}=f\left(x_{t}, a_{t}\right)$. For every $\phi \in$ $\Phi_{t}\left(a_{t}\right)$ such that $x_{t} \in \operatorname{supp}(\phi)$ :

(i) Update the visits counter: $N(\phi):=N(\phi)+1$.

(ii) If $\phi$ is visited for the first time, compute the model for this kernel. Namely,

(a) Compute the empirical reward and transition according to (11) and (12).

(b) Compute the upper reward value

$$
\tilde{r}(\phi):=\hat{r}(\phi)+\alpha \Delta(\phi),
$$

(c) Compute the transition uncertainty set

$$
\mathrm{UI}_{f}(\phi):=\{y \in \mathbb{X}: d(y, \hat{f}(\phi)) \leq \beta \Delta(\phi)\}
$$

\subsection{Splitting method}

Assume that a fixed uniform splitting scheme is used throughout (cf. Definition 3). Define a count threshold $\mathcal{N}$. We will split a kernel if the number of visits in its support exceeds $\mathcal{N}$. Now, let $\phi \in \Phi_{t}(a)$ be a parent kernel which is split at time $t$ into $K$ new kernels $\phi_{1}, \ldots, \phi_{K} \in \Phi_{t+1}(a)$, and denote by $(x, a, \hat{r}(\phi), \hat{f}(\phi))$ the sample of the parent kernel, with $x \in \operatorname{supp}(\phi), \hat{r}(\phi)=r(x, a), \hat{f}(\phi)=f(x, a)$. We initialize the model of the new kernels $\left\{\phi_{j}\right\}$ as follows:

1. If $x \in \operatorname{supp}\left(\phi_{j}\right)$, let $\phi_{j}$ inherit the sample of the parent kernel. Namely, set

$$
\begin{aligned}
\tilde{r}\left(\phi_{j}\right) & =\hat{r}(\phi)+\alpha \Delta\left(\phi_{j}\right), \\
\mathrm{UI}_{f}\left(\phi_{j}\right) & =\left\{y \in \mathbb{X}: d(y, \hat{f}(\phi)) \leq \beta \Delta\left(\phi_{j}\right)\right\} .
\end{aligned}
$$

2. Otherwise, initialize the uncertainty parameters to those of the parent kernel:

$$
\begin{aligned}
\tilde{r}\left(\phi_{j}\right) & =\tilde{r}(\phi), \\
\mathrm{UI}_{f}\left(\phi_{j}\right) & =\mathrm{UI}_{f}(\phi) .
\end{aligned}
$$

We note that this choice of the initialization leads to an improvement of the uncertainty parameters of the new kernels which contain the sample of the parent kernel. Moreover, if the whole history of samples is saved in the course of learning, we can further improve step (2) above by using corresponding samples to initialize the model of the new kernels.

In addition to this splitting criterion, we also employ a "stop-splitting" rule, based on the kernel support diameter. Let $\Delta_{\epsilon}$ be a (small) size threshold parameter. Then, if a kernel $\phi$ satisfies $\Delta(\phi) \leq \Delta_{\epsilon}$, it will not be split anymore. Since the number of times that the algorithm encounters a kernel with $\Delta(\phi)>\Delta_{\epsilon}$ can be bounded, it follows that the number of different stationary policies that the algorithm computes and uses can also be bounded. This will eventually enable us to prove a bound on the policy-mistake count, in Sect. 7.

Now, under a fixed uniform splitting scheme (Definition 3), we denote by $\Phi_{\epsilon}$ the coarsest achievable kernel set with $\Delta(\phi) \leq \Delta_{\epsilon}$ for all $\phi \in \Phi_{\epsilon}$. We call this set $\Delta_{\epsilon}$-supported kernel set. The number of kernels in $\Phi_{\epsilon}$ can be bounded as follows (see Sect. 7.4 for a proof). 
Algorithm 4 Splitting Algorithm

1. Initialize $\Phi_{t+1}(a)=\Phi_{t}(a)$, for all $a \in \mathbb{A}$.

2. For each $a \in \mathbb{A}$ and $\phi \in \Phi_{t}(a)$ which satisfy $N(\phi) \geq \mathcal{N}$ and $\Delta(\phi)>\Delta_{\epsilon}$, perform the following:

(a) Split this kernel according to the given uniform splitting scheme. Let $\phi_{1}, \ldots, \phi_{K} \in$ $\Phi_{t+1}(a)$ be the resulting sub-kernels after this split.

(b) Initialize the reward upper uncertainty bounds of the kernels:

$$
\tilde{r}\left(\phi_{j}\right)= \begin{cases}\hat{r}(\phi)+\alpha \Delta\left(\phi_{j}\right), & \text { if } \phi_{j} \text { contains the sample of the parent kernel } \phi, \\ \tilde{r}(\phi), & \text { otherwise. }\end{cases}
$$

(c) Initialize the transition uncertainty sets of the new kernels:

$$
\mathrm{UI}_{f}\left(\phi_{j}\right)=\left\{\begin{array}{l}
\left\{y \in \mathbb{X}: d(y, \hat{f}(\phi)) \leq \beta \Delta\left(\phi_{j}\right)\right\}, \\
\text { if } \phi_{j} \text { contains the sample of the parent kernel } \phi, \\
\mathrm{UI}_{f}(\phi), \text { otherwise. }
\end{array}\right.
$$

(d) Update the counts of the new cells as follows:

$$
N\left(\phi_{j}\right)= \begin{cases}1, & \text { if } \phi_{j} \text { contains the sample of the parent kernel } \phi \\ 0, & \text { otherwise. }\end{cases}
$$

Lemma 4 For a fixed uniform splitting scheme, with parameters $K$ and $\lambda$, and a single initial grid kernel set $\Phi_{0}$, we have that

$$
N_{\epsilon} \triangleq\left|\Phi_{\epsilon}\right| \leq\left|\Phi_{0}\right| K\left(\frac{\Delta_{\max }}{\Delta_{\epsilon}}\right)^{\log _{1 / \lambda}(K)}
$$

where $\Delta_{\max }$ is the diameter of the state space (from Assumption 1).

We summarize the splitting process in Algorithm 4. This completes the description of the ARL algorithm as outlined in Algorithm 1.

\section{UVF computation procedure}

In this section we propose a general procedure for solving the fixed point equation (13). Recall the definition of the upper value DP operator $\mathcal{T}$ (Definition 6). Lemma 2 shows that this operator is a contraction mapping and, in principle, the solution $\widetilde{V}_{t}$ of the fixed point equation (13) can be found using value iteration. Now, since this solution is defined on the infinite state space $\mathbb{X}$, a straightforward application of value iteration is infeasible. However, a more careful examination of Definition 6 reveals that in fact only the following finite set of target values is needed in order to compute the value $\widetilde{V}_{t}$ and the policy $\pi_{t}$ at any $x \in \mathbb{X}$ :

$$
\widetilde{T}(i, a) \triangleq \min _{y \in \mathrm{UI}_{f}\left(\phi_{i}^{a}\right)} \widetilde{V}_{t}(y), \quad a \in \mathbb{A}, \quad \phi_{i}^{a} \in \Phi_{t}(a), \quad i=1, \ldots,\left|\Phi_{t}(a)\right| .
$$

Indeed, we can rewrite (13) in terms of $\{\widetilde{T}(i, a)\}$ as follows: 


$$
\widetilde{V}_{t}(x)=\max _{a \in \mathbb{A}}\left\{\sum_{i=1}^{\left|\Phi_{t}(a)\right|} \phi_{i}^{a}(x)\left(\tilde{r}\left(\phi_{i}^{a}\right)+\gamma[\tilde{T}(i, a)+\bar{\omega}(i, a)]\right)\right\},
$$

where

$$
\bar{\omega}(i, a) \triangleq \bar{\omega}\left(2 \beta \Delta\left(\phi_{i}^{a}\right)\right) .
$$

This implies that

$$
\widetilde{T}(i, a)=\min _{x \in \mathrm{UI}_{f}\left(\phi_{i}^{a}\right)} \max _{a^{\prime} \in \mathbb{A}}\left\{\sum_{j=1}^{\left|\Phi_{t}\left(a^{\prime}\right)\right|} \phi_{j}^{a^{\prime}}(x)\left(\tilde{r}\left(\phi_{j}^{a^{\prime}}\right)+\gamma\left[\tilde{T}\left(j, a^{\prime}\right)+\bar{\omega}\left(j, a^{\prime}\right)\right]\right)\right\},
$$

for all $a \in \mathbb{A}, \phi_{i}^{a} \in \Phi_{t}(a), i=1, \ldots,\left|\Phi_{t}(a)\right|$.

Finally, we can show (using the same arguments as in proof of Lemma 2) that the operator associated with (20) is a contraction mapping (with contraction factor $\gamma$ ), so that the target values (19) can be computed using the following value iteration procedure:

1. Initialize $\widetilde{T}^{(0)}(i, a) \equiv V_{\max }$.

2. At iteration $k=1,2, \ldots$, update:

$$
\widetilde{T}^{(k)}(i, a)=\min _{x \in \mathrm{UI}_{f}\left(\phi_{i}^{a}\right)} \max _{a^{\prime} \in \mathbb{A}}\left\{\sum_{j=1}^{\left|\Phi_{t}\left(a^{\prime}\right)\right|} \phi_{j}^{a^{\prime}}(x)\left(\tilde{r}\left(\phi_{j}^{a^{\prime}}\right)+\gamma\left[\widetilde{T}^{(k-1)}\left(j, a^{\prime}\right)+\bar{\omega}\left(j, a^{\prime}\right)\right]\right)\right\} .
$$

Unfortunately, in order to carry out the above procedure, one in principle must solve an infinite number of minmax problems (21) of a not necessarily convex function. This is of course infeasible in general. In Sect. 8 we will present an incremental variant of our algorithm, in which only few iterations are performed at each time step. Note that this still leaves us with the need of solving the minmax problem above. However, at least in the special cases of constant and triangular kernels, this procedure can be practically implemented. See Appendix for details.

\section{Main results}

We next summarize the main results regarding our algorithm. Proofs are deferred to the next section.

Recall the definition of the policy-mistake count (4) and the corresponding nearoptimality criterion. Also, recall that $\Phi_{\epsilon}$ is the coarsest achievable kernel set with $\Delta(\phi) \leq$ $\Delta_{\epsilon}$, and satisfies Lemma 4. First we present the main theorem, which provides a mistake bound of our scheme in terms of the number of kernels in $\Phi_{\epsilon}$.

Theorem 1 Let $\epsilon>0$ be given and assume that the ARL algorithm's input parameter $\Delta_{\epsilon}$ is chosen such that

$$
\frac{2 \alpha \Delta_{\epsilon}+\gamma \bar{\omega}\left(2 \beta \Delta_{\epsilon}\right)}{1-\gamma} \leq \frac{\epsilon}{2} .
$$

Then, the policy-mistake count of the algorithm is bounded by

$$
\operatorname{PMC}(\epsilon) \leq \frac{\left|\Phi_{\epsilon}\right||\mathbb{A}|(\mathcal{N}+1)}{1-\gamma} \ln \frac{2 V_{b}}{\epsilon},
$$

where $\mathcal{N}$ is the count threshold parameter. 
Condition (22) does not provide an explicit condition on the algorithm's input $\Delta_{\epsilon}$. However, such condition can be obtained, at least in case $\gamma \beta \neq 1$, using the particular choice of MCB $\bar{\omega}$ presented in Lemma 1.

Lemma 5 Suppose that $\gamma \beta \neq 1$. Then, the precondition (22) of Theorem 1 is satisfied under the following assumptions.

1. If $\gamma \beta<1$, let

$$
\Delta_{\epsilon}=\frac{(1-\gamma)(1-\gamma \beta)}{4 \alpha} \epsilon
$$

2. If $\gamma \beta>1$, let

$$
\Delta_{\epsilon}=\left(\frac{(1-\gamma) \epsilon}{2\left(2 \alpha+\gamma c(2 \beta)^{\log _{\beta}(1 / \gamma)}\right)}\right)^{\log _{1 / \gamma} \beta},
$$

where $c$ is defined in (9).

Remark Using Lemmas 4 and 5, one can obtain an explicit dependence of the mistake bound on $\epsilon$ (and other parameters). See the "Discussion" subsection below for a further elaboration on the nature of the bounds for $\gamma \beta<1$ and $\gamma \beta>1$.

As a corollary of Theorem 1, we obtain the following result which is of independent interest.

Theorem 2 Consider the non-adaptive learning algorithm which uses the $\Delta_{\epsilon}$-supported kernel set $\Phi_{t}(a)=\Phi_{\epsilon}$ for all $t \geq 0$ and $a \in \mathbb{A}$, with $\Delta_{\epsilon}$ satisfying (22). Then, the policymistake count of the non-adaptive algorithm is bounded by

$$
\operatorname{PMC}(\epsilon) \leq \frac{\left|\Phi_{\epsilon}\right||\mathbb{A}|}{1-\gamma} \ln \frac{2 V_{b}}{\epsilon}
$$

Proof Follows immediately from Theorem 1, by considering the ARL algorithm (Algorithm 1) initialized to $\Phi_{0}(a) \equiv \Phi_{\epsilon}$ and using $\mathcal{N}=0$.

In addition to Theorem 1, we can obtain a possibly tighter mistake bound in terms of the posterior number of kernels actually used in the course of the algorithm. In fact, the purpose of adaptive-resolution approximation is that as time progresses, the algorithm will refine kernels only in the vicinity of the optimal trajectory. Therefore, the actual number of kernels in the long term should be much less than $\left|\Phi_{\epsilon}\right|$. We make this more formal below.

Definition 7 Let $x_{0}$ be the initial state and let $N_{\infty}\left(x_{0}, a\right)$ be the number of cells in the grid $\Phi_{t}(a)$ as $t \rightarrow \infty$, that is

$$
N_{\infty}\left(x_{0}, a\right) \triangleq \lim _{t \rightarrow \infty}\left|\Phi_{t}(a)\right|
$$

Also, let $N_{\infty}\left(x_{0}\right) \triangleq \sum_{a \in \mathbb{A}} N_{\infty}\left(x_{0}, a\right)$. 
We note that the limit in (25) exists and is finite, since $\left|\Phi_{t}(a)\right|$ increases in $t$, while $\left|\Phi_{t}(a)\right| \leq\left|\Phi_{\epsilon}\right|$ due to the enforced "stop-splitting" rule. For the same reason, we have the trivial bound $N_{\infty}\left(x_{0}\right) \leq\left|\Phi_{\epsilon}\right||\mathbb{A}|$.

Theorem 3 Let $\epsilon>0$ and assume that the ARL algorithm receives an input parameter $\Delta_{\epsilon}$ as in Theorem 1. Then, it holds that

$$
\operatorname{PMC}(\epsilon) \leq \frac{2 N_{\infty}\left(x_{0}\right) \mathcal{N}}{1-\gamma} \ln \frac{2 V_{b}}{\epsilon}
$$

Remark The bound of Theorem 3 becomes better than that of Theorem 1 if $N_{\infty}\left(x_{0}\right) \leq$ $\frac{1}{2}\left|\Phi_{\epsilon}\right||\mathbb{A}|$.

\section{Discussion}

First, consider the contractive case $(\gamma \beta<1)$. Theorem 1 implies that the mistake bound is linear in $\left|\Phi_{\epsilon}\right|$. Therefore, using Lemma 4 and (23), we obtain the following explicit dependence on $\epsilon$ (ignoring the log factor):

$$
\operatorname{PMC}(\epsilon) \lesssim C(1 / \epsilon)^{n}|\mathbb{A}|(\mathcal{N}+1),
$$

where the constant $C$ is polynomial in $\alpha, 1 /(1-\gamma)$ and in $1 /(1-\gamma \beta)$. Note that the exponential dependence on the dimension $n$ of the state-space is an obvious artifact of the dense aggregation approach, since in most cases $\log _{1 / \lambda}(K)$, which appears in Lemma 4 , is of order of $n$.

In the expansive case $(\gamma \beta>1)$, the analysis becomes more involved. In particular, it is shown in Sect. 7 that to obtain the mistake bounds of Theorems 1 and 3, the size threshold should be taken as in (24). As a result, in the expansive case we obtain a worse explicit dependence of the mistake bound on $\epsilon$, as follows:

$$
\operatorname{PMC}(\epsilon) \lesssim C^{\prime}(1 / \epsilon)^{n \log _{1 / \gamma} \beta}|\mathbb{A}|(\mathcal{N}+1)
$$

where $C^{\prime}$ is polynomial in $\alpha, \beta, 1 /(1-\gamma)$, and exponential in $\log _{1 / \gamma} \beta$. Note that in this case $\log _{1 / \gamma} \beta>1$.

In the context of the posterior bound (Theorem 3), it should be noted that there is a trade-off between the choice of the count threshold $\mathcal{N}$ and the number of kernels at infinity $N_{\infty}\left(x_{0}\right)$. If we choose $\mathcal{N}$ too small, the algorithm will perform many splits, and consequently $N_{\infty}\left(x_{0}\right)$ will be large. In this case it may happen that the algorithm will produce redundant kernels, which are not actually needed for near-optimal performance. On the other hand, if we choose large $\mathcal{N}$, the algorithm will perform less splits, resulting in a smaller $N_{\infty}\left(x_{0}\right)$. This however may lead to a slower convergence to the optimal trajectory.

Two comparisons that may be of interest follow. First, consider a standard exploration algorithm (such as the R-MAX, $E^{3}$, or MBIE algorithms mentioned above) applied to a fixed-resolution model with a sufficiently fine kernel set $\Phi_{\epsilon}$ over the state space, without using the uncertainty intervals employed in our algorithm. In particular, once the reward is obtained for a given aggregated state-action pair, this naïve algorithm would treat it as a correct reward, despite the fact that the actual reward (at some true state that falls in the aggregated one) might be higher. As a result, the computed value function might underestimate the optimal one, and consequently no formal guarantees can be provided for such algorithm. 
Moreover, consider our algorithm applied to a fixed-resolution model, where a sufficiently fine kernel set $\Phi_{\epsilon}$ is used from the outset (that is, $\Phi_{0}=\Phi_{\epsilon}$ ). As Theorem 2 shows, the mistake bound in such case will be $\mathcal{N}+1$ times better than that of Theorem 1 . However, such an algorithm is not feasible if $\left|\Phi_{\epsilon}\right|$ is large.

\section{Analysis of the ARL algorithm}

Below is the outline of the analysis. First we show that there exists an MCB (Definition 5), which will justify Definition 6. Then, we show that there exists a unique solution to (13), and that this solution upper bounds the optimal value $V$. Finally, we prove that under certain conditions on the kernel sets, the optimal policy with respect to the UVF (16) is an $\epsilon$-optimal policy, which will enable us to prove a polynomial bound on the policy-mistake count of the algorithm.

To simplify the notation, throughout the analysis we write UI for the transition uncertainty set (instead of $\mathrm{UI}_{f}$ ).

\subsection{Continuity of the optimal value function}

In this subsection we prove Lemma 1. In particular, we show that under continuity Assumption 2, the optimal value function is also uniformly continuous. However, it will be Lipschitz continuous, only when $\gamma \beta<1$. Otherwise, a weaker sort of continuity, namely Hölder continuity, holds.

Proof of Lemma 1 Fix $x_{1}, x_{2} \in \mathbb{X}$. From the optimality equation (2), we have that

$$
\begin{aligned}
\left|V\left(x_{1}\right)-V\left(x_{2}\right)\right| & \leq \max _{a}\left|r\left(x_{1}, a\right)-r\left(x_{2}, a\right)\right|+\gamma \max _{a}\left|V\left(f\left(x_{1}, a\right)\right)-V\left(f\left(x_{2}, a\right)\right)\right| \\
& \leq \alpha d\left(x_{1}, x_{2}\right)+\gamma \max _{a}\left|V\left(f\left(x_{1}, a\right)\right)-V\left(f\left(x_{2}, a\right)\right)\right|,
\end{aligned}
$$

where the second inequality follows by Assumption 2. Also by this assumption, we have that

$$
d\left(f\left(x_{1}, a\right), f\left(x_{2}, a\right)\right) \leq \beta d\left(x_{1}, x_{2}\right),
$$

for any $a$. Applying the above inequalities iteratively, for any integer $H>0$, we obtain the following bound

$$
\left|V\left(x_{1}\right)-V\left(x_{2}\right)\right| \leq \alpha d\left(x_{1}, x_{2}\right) \sum_{k=0}^{H-1}(\gamma \beta)^{k}+\gamma^{H} V_{b} .
$$

First, consider the case of $\gamma \beta<1$. Here we can take $H=\infty$ in the above bound, and obtain the desired result. Next, if $\gamma \beta>1$, we have that

$$
\begin{aligned}
\left|V\left(x_{1}\right)-V\left(x_{2}\right)\right| & \leq \alpha d\left(x_{1}, x_{2}\right) \frac{(\gamma \beta)^{H}-1}{\gamma \beta-1}+\gamma^{H} V_{b} \\
& \leq \alpha d\left(x_{1}, x_{2}\right) \frac{(\gamma \beta)^{H}}{\gamma \beta-1}+\gamma^{H} V_{b} .
\end{aligned}
$$


Now, since $\gamma<1$, there exist a minimal $H=H_{0}\left(x_{1}, x_{2}\right)$, such that

$$
\gamma^{H_{0}} V_{b} \leq \alpha d\left(x_{1}, x_{2}\right) \frac{(\gamma \beta)^{H_{0}}}{\gamma \beta-1},
$$

implying that

$$
\left|V\left(x_{1}\right)-V\left(x_{2}\right)\right| \leq 2 \alpha d\left(x_{1}, x_{2}\right) \frac{(\gamma \beta)^{H_{0}}}{\gamma \beta-1} .
$$

Since $H_{0}$ is the minimal $H$ satisfying (27), it holds that

$$
\alpha d\left(x_{1}, x_{2}\right) \frac{\beta^{H_{0}-1}}{\gamma \beta-1}<V_{b} \leq \alpha d\left(x_{1}, x_{2}\right) \frac{\beta^{H_{0}}}{\gamma \beta-1},
$$

implying that $\beta^{H_{0}}<\frac{V_{b} \beta(\gamma \beta-1)}{\alpha d\left(x_{1}, x_{2}\right)}, \beta^{H_{0}} \geq \frac{V_{b}(\gamma \beta-1)}{\alpha d\left(x_{1}, x_{2}\right)}$, and consequently,

$$
\gamma^{H_{0}} \leq\left(\frac{V_{b}(\gamma \beta-1)}{\alpha d\left(x_{1}, x_{2}\right)}\right)^{\log _{\beta} \gamma} .
$$

Therefore, we have that

$$
(\gamma \beta)^{H_{0}} \leq \beta\left(\frac{V_{b}(\gamma \beta-1)}{\alpha d\left(x_{1}, x_{2}\right)}\right)^{\log _{\beta} \gamma \beta} .
$$

Substituting (29) in (28) yields the result.

7.2 The upper value function

Below we prove Lemmas 2 and 3. That is, we show that the operator $\mathcal{T}$ (Definition 6) is a contraction operator, and that the solution of (13) is indeed an upper bound on the optimal value function.

Proof of Lemma 2 Given two functions $g_{1}$ and $g_{2}$, we have the following sequence of inequalities for any $x \in \mathbb{X}$ :

$$
\begin{aligned}
\left|\left(\mathcal{T} g_{1}\right)(x)-\left(\mathcal{T} g_{2}\right)(x)\right| & \leq \gamma \max _{a \in \mathbb{A}}\left|\sum_{\phi \in \Phi_{t}(a)} \phi(x)\left(\min _{y \in \mathrm{UI}(\phi)} g_{1}(y)-\min _{y \in \mathrm{UI}(\phi)} g_{2}(y)\right)\right| \\
& \leq \gamma \max _{a \in \mathbb{A}} \sum_{\phi \in \Phi_{t}(a)} \phi(x) \max _{y \in \mathrm{UI}(\phi)}\left|g_{1}(y)-g_{2}(y)\right| \\
& \leq \gamma \max _{y \in \mathbb{X}}\left|g_{1}(y)-g_{2}(y)\right| \sum_{\phi \in \Phi_{t}(a)} \phi(x) \\
& =\gamma \max _{y \in \mathbb{X}}\left|g_{1}(y)-g_{2}(y)\right| \triangleq \gamma\left\|g_{1}-g_{2}\right\|_{\infty},
\end{aligned}
$$

where the first inequality follows by Definition 6 and the equality follows by the definition of kernel set (Definition 2). Hence, $\left\|\mathcal{T} g_{1}-\mathcal{T} g_{2}\right\|_{\infty} \leq \gamma\left\|g_{1}-g_{2}\right\|_{\infty}$, which proves the result.

Proof of Lemma 3 Since, by Lemma 2, $\mathcal{T}$ is a contraction operator, we can prove the claim by induction on the steps of value iteration. For the base case, let $\widetilde{V}^{0}(x)=V_{\max } \geq$ 
$V(x), \forall x \in \mathbb{X}$. Now assume that the claim holds for the $n$-th iteration. For the $n+1$-th iteration we have by the Lipschitz continuity of the reward (Assumption 2) and by the definition of $\tilde{r}(\phi)$, that for all $a \in \mathbb{A}, \phi \in \Phi(a)$, and $x \in \operatorname{supp}(\phi)$

$$
\tilde{r}(\phi)=r\left(x_{s}, a\right)+\alpha \Delta(\phi) \geq r(x, a),
$$

where $x_{s}$ is a sample point of $\phi$. Therefore, by the fact that $\sum_{\phi \in \Phi(a)} \phi(x)=1$ for all $x$ (Definition 2),

$$
\sum_{\phi \in \Phi(a)} \phi(x) \tilde{r}(\phi) \geq r(x, a), \quad \forall x \in \mathbb{X}, a \in \mathbb{A} .
$$

Now, set $y^{*}=\min _{y \in \mathrm{UI}(\phi)} \widetilde{V}^{n}(y)$. For any $x \in \operatorname{supp}(\phi)$, by Assumption 2 and by the definition of $\mathrm{UI}(\phi)$, we have that $f(x, a) \in \mathrm{UI}(\phi)$, implying that

$$
d\left(y^{*}, f(x, a)\right) \leq 2 \beta \Delta(\phi) .
$$

Therefore, by the definition of MCB (Definition 5), $\left|V\left(y^{*}\right)-V(f(x, a))\right| \leq \bar{\omega}(2 \beta \Delta(\phi))$. Consequently,

$$
\widetilde{V}^{n}\left(y^{*}\right)+\bar{\omega}(2 \beta \Delta(\phi)) \geq V\left(y^{*}\right)+\bar{\omega}(2 \beta \Delta(\phi)) \geq V(f(x, a)),
$$

where the first inequality follows by the induction assumption. We thus have shown that

$$
\sum_{\phi \in \Phi(a)} \phi(x)\left(\min _{y \in \mathrm{UI}(\phi)} \widetilde{V}^{n}(y)+\bar{\omega}(2 \beta \Delta(\phi))\right) \geq V(f(x, a)), \quad \forall x \in \mathbb{X}, a \in \mathbb{A} .
$$

Combining (30) and (31) we obtain that

$$
\begin{aligned}
\widetilde{V}^{n+1}(x) & =\max _{a \in \mathbb{A}}\left\{\sum_{\phi \in \Phi(a)} \phi(x) \tilde{r}(\phi)+\gamma \sum_{\phi \in \Phi(a)} \phi(x)\left(\min _{y \in \mathrm{UI}(\phi)} \widetilde{V}^{n}(y)+\bar{\omega}(2 \beta \Delta(\phi))\right)\right\} \\
& \geq \max _{a \in \mathbb{A}}\{r(x, a)+\gamma V(f(x, a))\}=V(x),
\end{aligned}
$$

which completes the induction proof. Since $\widetilde{V}^{n} \rightarrow \widetilde{V}$, the result follows.

\subsection{Near-optimality of the UVF optimal policy}

In this section we provide a sufficient condition on the diameter of the support of kernels, which ensures that the return obtained by the policy $\mathcal{A}_{t}=\left\{\pi_{\tau}\right\}_{\tau=t}^{\infty}$ which the algorithm implements at time $t$, is $\epsilon$-close to the UVF: $\widetilde{V}_{t}(x)-J_{M}^{\mathcal{A}_{t}}(x) \leq \epsilon$. This will imply that $V(x)-J_{M}^{\mathcal{A}_{t}}(x) \leq \epsilon$, since $\widetilde{V}_{t}$ is an upper bound on the optimal value; namely, this will imply that $\mathcal{A}_{t}$ is an $\epsilon$-optimal policy from $x$.

The following is a standard definition, which specifies a mixing time of any stationary policy in discounted MDPs.

Definition $8(\epsilon / 2$-Horizon Time) In an MDP $M$, the $\epsilon / 2$-horizon time is given by

$$
T_{\epsilon / 2} \triangleq \log _{1 / \gamma} \frac{2 V_{b}}{\epsilon} .
$$


To proceed, we introduce the definitions of known kernel-action pairs and the escape event.

Definition 9 (Known Pairs) At any time $t$, define the set of known kernel-action pairs:

$$
\mathcal{K}_{t} \triangleq\left\{(\phi, a) \in \Phi_{t}(a) \times \mathbb{A}: \Delta(\phi) \leq \Delta_{\epsilon}, \phi \text { was previously sampled }\right\}
$$

Definition 10 (Escape Event) At any time $t$, define the escape event from a given starting state $x \in \mathbb{X}$ :

$$
\begin{aligned}
E_{t}(x) \triangleq & \left\{\text { In a trial generated by starting from state } x \text { and following } \mathcal{A}_{t}\right. \\
& \text { for } T_{\epsilon / 2} \text { steps in } M, \text { a pair }\left(x_{t}, a_{t}\right) \text { is encountered, such that } x_{t} \in \operatorname{supp}(\phi), \\
& \text { but } \left.\left(\phi, a_{t}\right) \notin \mathcal{K}_{t}\right\} .
\end{aligned}
$$

Definition 11 (Episode) An episode is a maximal period of time $\left[t_{0}, t_{1}\right]$, in which:

(i) All visited state-action pairs $\left(x_{t}, a_{t}\right)$ at times $t=t_{0}, \ldots, t_{1}-1$ satisfy that for each kernel $\phi \in \Phi_{t}\left(a_{t}\right)$ with $x_{t} \in \operatorname{supp}(\phi)$, it holds that

(a) $\Delta(\phi) \leq \Delta_{\epsilon}$,

(b) $\phi$ was previously sampled (that is, was previously visited).

(ii) At time $t=t_{1}$, the algorithm encounters a pair for which the condition in (i) is not true.

Note that during each episode, a fixed stationary policy is used by the algorithm, and the policy is (potentially) changed only at the beginning of each episode. Also, observe that the above definitions depend on the size threshold parameter $\Delta_{\epsilon}$. The next lemma formulates the condition on $\Delta_{\epsilon}$ which will imply that the execution of the algorithm's implemented policy $\mathcal{A}_{t}$ from time $t$ will obtain a return which is $\epsilon$-close to the UVF.

Lemma 6 Let $\epsilon>0$ be given and assume that $\Delta_{\epsilon}$ is chosen such that

$$
\frac{1}{1-\gamma}\left[2 \alpha \Delta_{\epsilon}+\gamma \bar{\omega}\left(2 \beta \Delta_{\epsilon}\right)\right] \leq \frac{\epsilon}{2}
$$

Then,

$$
\widetilde{V}_{t}(x)-J_{M}^{\mathcal{A}_{t}}(x) \leq \epsilon+\mathbb{I}\left\{E_{t}(x)\right\} V_{b}
$$

holds for all $t$ and $x \in \mathbb{X}$.

Proof At given time $t_{0}$, we consider the execution of the (non-stationary) policy $\mathcal{A}_{t_{0}}$ for $T_{\epsilon / 2}$ time steps in $M$. Now, we have two mutually exclusive cases:

(a) For every $\left(x_{t}, a_{t}\right)$ it holds that for each kernel $\phi \in \Phi_{t}\left(a_{t}\right)$ with $x_{t} \in \operatorname{supp}(\phi), \Delta(\phi) \leq \Delta_{\epsilon}$ and $\phi$ was sampled.

(b) There exists at least one $t \in\left[t_{0}, t_{0}+T_{\epsilon / 2}-1\right]$ such that the above condition does not hold.

The case (b) above is easy-if it happens, we have that a pair $(\phi, a)$ not in $\mathcal{K}_{t_{0}}$ is encountered. Thus, the escape event $E_{t_{0}}\left(x_{t_{0}}\right)$ occurred during the execution of $\mathcal{A}_{t_{0}}$ for $T_{\epsilon / 2}$ time-steps, which is expressed by the $\mathbb{I}\left\{E_{t_{0}}\left(x_{t_{0}}\right)\right\} V_{b}$ term in the bound. 
Now, if (a) is the case, during the execution of $\mathcal{A}_{t_{0}}$ for $T_{\epsilon / 2}$ time steps we stay in the same episode (Definition 11), and thus the algorithm's policy remains unchanged and it is the stationary policy $\pi_{t_{0}}$. For simplicity, assume $t_{0}=0$, write $\pi$ for $\pi_{0}$ and $\widetilde{V}$ for $\widetilde{V}_{0}$, and recall that $\pi$ is the greedy policy with respect to $\widetilde{V}(16)$. Thus,

$$
\widetilde{V}\left(x_{0}\right)=\sum_{\phi \in \Phi\left(a_{0}\right)} \phi\left(x_{0}\right) \tilde{r}(\phi)+\gamma \sum_{\phi \in \Phi\left(a_{0}\right)} \phi\left(x_{0}\right)\left(\min _{y \in \mathrm{UI}(\phi)} \tilde{V}(y)+\bar{\omega}(2 \beta \Delta(\phi))\right) .
$$

Also, by Bellman's equation,

$$
J_{M}^{\pi}\left(x_{0}\right)=r\left(x_{0}, a_{0}\right)+\gamma J_{M}^{\pi}\left(x_{1}\right) .
$$

Now, we have that

$$
\begin{aligned}
\tilde{V}\left(x_{0}\right)-J_{M}^{\pi}\left(x_{0}\right) & \leq 2 \alpha \Delta_{\epsilon}+\gamma\left(\sum_{\phi \in \Phi\left(a_{0}\right)} \phi\left(x_{0}\right)\left(\min _{y \in \mathrm{UI}(\phi)} \tilde{V}(y)+\bar{\omega}(2 \beta \Delta(\phi))\right)-J_{M}^{\pi}\left(x_{1}\right)\right) \\
& \leq 2 \alpha \Delta_{\epsilon}+\gamma\left(\left(\widetilde{V}\left(x_{1}\right)+\bar{\omega}\left(2 \beta \Delta_{\epsilon}\right)\right)\left[\sum_{\phi \in \Phi\left(a_{0}\right)} \phi\left(x_{0}\right)\right]-J_{M}^{\pi}\left(x_{1}\right)\right) \\
& =2 \alpha \Delta_{\epsilon}+\gamma \bar{\omega}\left(2 \beta \Delta_{\epsilon}\right)+\gamma\left(\widetilde{V}\left(x_{1}\right)-J_{M}^{\pi}\left(x_{1}\right)\right) .
\end{aligned}
$$

Here, inequality (33) follows by the continuity assumption on the reward, since for every kernel $\phi \in \Phi\left(a_{0}\right)$ with $x_{0} \in \operatorname{supp}(\phi)$ we have that

$$
\begin{aligned}
\tilde{r}(\phi)-r\left(x_{0}, a_{0}\right) & =r\left(x^{\prime}, a_{0}\right)+\alpha \Delta(\phi)-r\left(x_{0}, a_{0}\right) \\
& \leq 2 \alpha \Delta(\phi) \leq 2 \alpha \Delta_{\epsilon},
\end{aligned}
$$

where $x^{\prime}$ is the sample that was received for this kernel, and the last inequality is due to the fact that we are within an episode. Inequality (34) holds since $x_{1} \in \mathrm{UI}(\phi)$ for every $\phi \in \Phi\left(a_{0}\right)$ with $x_{0} \in \operatorname{supp}(\phi)$. Thus, proceeding iteratively, we obtain the following bound:

$$
\tilde{V}\left(x_{0}\right)-J_{M}^{\pi}\left(x_{0}\right) \leq \sum_{t=0}^{T_{\epsilon / 2}-1} \gamma^{t}\left[2 \alpha \Delta_{\epsilon}+\gamma \bar{\omega}\left(2 \beta \Delta_{\epsilon}\right)\right]+\gamma^{T_{\epsilon / 2}} V_{b} .
$$

By the definition of $T_{\epsilon / 2}$ (Definition 8), we have $\gamma^{T_{\epsilon / 2}} V_{b} \leq \frac{\epsilon}{2}$.

Now, the first term above can be trivially bounded as follows:

$$
\begin{aligned}
\sum_{t=0}^{T_{\epsilon / 2}-1} \gamma^{t}\left[2 \alpha \Delta_{\epsilon}+\gamma \bar{\omega}\left(2 \beta \Delta_{\epsilon}\right)\right] & \leq \sum_{t=0}^{\infty} \gamma^{t}\left[2 \alpha \Delta_{\epsilon}+\gamma \bar{\omega}\left(2 \beta \Delta_{\epsilon}\right)\right] \\
& =\frac{1}{1-\gamma}\left[2 \alpha \Delta_{\epsilon}+\gamma \bar{\omega}\left(2 \beta \Delta_{\epsilon}\right)\right] \leq \frac{\epsilon}{2},
\end{aligned}
$$

where the last inequality follows by the hypothesis (32) of the lemma. This completes the proof.

To conclude this subsection, we prove Lemma 5, which provides an explicit condition on the algorithm's input $\Delta_{\epsilon}$. 
Proof of Lemma 5 If $\gamma \beta<1$, by the explicit expression of $\bar{\omega}$ (see Lemma 1), we have to check that if $\Delta_{\epsilon}$ satisfies (23), then

$$
\frac{1}{1-\gamma}\left[2 \alpha \Delta_{\epsilon}+\gamma \frac{\alpha}{1-\gamma \beta} 2 \beta \Delta_{\epsilon}\right] \leq \frac{\epsilon}{2}
$$

holds. This is indeed true, which can be easily verified by substitution.

Now, for $\gamma \beta>1$, by the explicit expression of $\bar{\omega}$ (Lemma 1), we have to check that

$$
2 \alpha \Delta_{\epsilon}+\gamma c\left(\Delta_{\epsilon} 2 \beta\right)^{\log _{\beta}(1 / \gamma)} \leq \frac{\epsilon(1-\gamma)}{2},
$$

holds, if $\Delta_{\epsilon}$ satisfies (24). Since $\log _{\beta}(1 / \gamma)<1$ in this case, the sufficient condition is ${ }^{4}$

$$
\left(\Delta_{\epsilon}\right)^{\log _{\beta}(1 / \gamma)}\left(2 \alpha+\gamma c(2 \beta)^{\log _{\beta}(1 / \gamma)}\right) \leq \frac{\epsilon(1-\gamma)}{2} .
$$

This last inequality is satisfied with equality, by substituting (24), and that completes the proof of the lemma.

\subsection{The cardinality of $\Delta_{\epsilon}$-supported kernel sets}

Before proving the mistake bounds, we prove Lemma 4. Namely, we provide an upper bound on the number of kernels $N_{\epsilon}=\left|\Phi_{\epsilon}\right|$.

Proof of Lemma 4 For every $\phi \in \Phi_{0}$, consider performing $k(\phi)$ splits iteratively, such that at each iteration we obtain new $K$ kernels instead of the original one. It follows that after $k(\phi)$ such splits, the size of a split kernel $\phi^{\prime}$ satisfies $\Delta\left(\phi^{\prime}\right) \leq \lambda^{k(\phi)} \Delta(\phi)$. In addition, the size of the kernel set that contains all such kernels $\phi^{\prime}$ is

$$
N=\sum_{\phi \in \Phi_{0}} K^{k(\phi)}
$$

Thus, for each $\phi \in \Phi_{0}$, we need to find the minimal $k(\phi)$, such that

$$
\lambda^{k(\phi)} \Delta(\phi) \leq \Delta_{\epsilon} .
$$

From (36), it follows that this minimal $k(\phi)=k^{*}(\phi)$ satisfies

$$
\log _{1 / \lambda}\left(\frac{\Delta(\phi)}{\Delta_{\epsilon}}\right) \leq k^{*}(\phi)<\log _{1 / \lambda}\left(\frac{\Delta(\phi)}{\Delta_{\epsilon}}\right)+1 .
$$

Substituting the last inequality in (35) yields

$$
\begin{aligned}
N_{\epsilon} & =\sum_{\phi \in \Phi_{0}} K^{k^{*}(s)} \leq K \sum_{\phi \in \Phi_{0}}(K)^{\log _{1 / \lambda}\left(\frac{\Delta(\phi)}{\Delta \epsilon}\right)} \\
& =K \sum_{\phi \in \Phi_{0}}\left(\frac{\Delta(\phi)}{\Delta_{\epsilon}}\right)^{\log _{1 / \lambda}(K)} \leq\left|\Phi_{0}\right| K\left(\frac{\Delta_{\text {max }}}{\Delta_{\epsilon}}\right)^{\log _{1 / \lambda}(K)},
\end{aligned}
$$

which completes the proof.

\footnotetext{
${ }^{4}$ This is true, of course, for sufficiently small $\epsilon$.
} 
Remark We note that Lemma 4 shows an exponential dependence of $N_{\epsilon}$ on the state space dimension $n$ since in most cases $\log _{1 / \lambda}(K)$ is of order of $n$.

\subsection{Proof of Theorem 1}

First, we note that the escape event $E_{t}(x)$ (Definition 10) can be viewed as an exploration event. If it occurs at some time $t \geq 0$, the algorithm will encounter (in an execution of length $T_{\epsilon / 2}$ ) a kernel $\phi$ which is not sampled and/or is not in $\Phi_{\epsilon}$ (that is, $\Delta(\phi)>\Delta_{\epsilon}$ ). This fact can be interpreted as a "discovery" of new information, since every such occurrence of an "unknown" kernel will lead to an increase of the count of the kernel, and, eventually, to the split of the kernel.

The next lemma shows that the number of times that the escape event can occur is bounded.

Lemma 7 The number of times that $E_{t}(x)$ can occur is bounded by $N_{\epsilon}|\mathbb{A}|(\mathcal{N}+1) T_{\epsilon / 2}$.

Proof Note that any kernel $\phi \in \Phi_{t}(a)$ for any $a$ and $t$, can be visited no more than $\mathcal{N}$ timesafter this number of times, the kernel is split. Now think of the kernel-based representation of the state space as a tree, with kernels as leaves. The internal nodes in such tree represent larger kernels, that were used in previous episodes. Now, the number of such internal nodes is less than or equal to the number of leaves, since the splitting coefficient is greater than or equal to 2. Using this tree representation, the visit to the "unknown" kernel can be interpreted as a visit to an internal node of $\Phi_{\epsilon}$. Since the counter of this kernel is incremented in this visit, by a simple counting argument (a.k.a. the Pigeonhole Principle), the number of times that the algorithm can encounter an internal node of $\Phi_{\epsilon}$ is bounded by

$$
\text { (number of internal nodes of } \left.\Phi_{\epsilon}\right) \cdot \mathcal{N} \cdot|\mathbb{A}| \leq N_{\epsilon} \mathcal{N}|\mathbb{A}| \text {. }
$$

Finally, when the algorithm encounters a leaf of $\Phi_{\epsilon}$, then only one such occurrence is sufficient in order to the kernel to become sampled. Again, by a simple counting argument, the number of times this can occur is bounded by

$$
\text { (number of leaves of } \left.\Phi_{\epsilon}\right) \cdot|\mathbb{A}|=N_{\epsilon}|\mathbb{A}| \text {. }
$$

To conclude, the number of times that an "unknown" kernel can be encountered is bounded by $N_{\epsilon} \mathcal{N}|\mathbb{A}|+N_{\epsilon}|\mathbb{A}|=N_{\epsilon}(\mathcal{N}+1)|\mathbb{A}|$.

At each time $t$, consider the execution of a (non-stationary) policy $\mathcal{A}_{t}$ for $T_{\epsilon / 2}$ time steps in $M$. We have two mutually exclusive cases:

(a) If starting at time $t$, we execute the policy $\mathcal{A}_{t}$ for $T_{\epsilon / 2}$ time steps, without encountering an "unknown" kernel (that is, a pair $(\phi, a)$ not in $\left.\mathcal{K}_{t}\right)$, there is no occurrence of the escape event $E_{t}(x)$.

(b) If starting at time $t$, we execute the policy $\mathcal{A}_{t}$ for $T_{\epsilon / 2}$ time steps, and encounter at least one unknown kernel at time $t \leq t^{\prime} \leq t+T_{\epsilon / 2}$, the escape event $E_{t}(x)$ occurs.

We then wish to bound the number of time steps that (b) is the case. In the worst case we will encounter an unknown kernel at the end of the execution episode of length $T_{\epsilon / 2}$. In this case, we have that all the succeeding executions for $t<t^{\prime} \leq t+T_{\epsilon / 2}$ will also encounter this unknown kernel. That is, if $E_{t}\left(x_{t}\right)$ occurs at some time $t$, also $E_{t^{\prime}}\left(x_{t^{\prime}}\right)$ for $t<t^{\prime} \leq t+T_{\epsilon / 2}$ will occur, in the worst case. Since after $N_{\epsilon}(\mathcal{N}+1)|\mathbb{A}|$ visits to unknown kernels, all the kernels will become known, $E_{t}(x)$ can occur at most $N_{\epsilon}(\mathcal{N}+1)|\mathbb{A}| T_{\epsilon / 2}$ times. 
Finally, we prove the main theorem regarding the mistake bound of the ARL algorithm.

Proof of Theorem 1 For each time $t$, we consider the execution of policy $\mathcal{A}_{t}$ for $T_{\epsilon / 2}$ timesteps in $M$, with the initial state in each such execution $x_{t}$. We then have that

$$
\begin{aligned}
J_{M}^{\mathcal{A}_{t}}\left(x_{t}\right) & \geq \widetilde{V}_{t}\left(x_{t}\right)-\epsilon-\mathbb{I}\left\{E_{t}\left(x_{t}\right)\right\} V_{b} \\
& \geq V\left(x_{t}\right)-\epsilon-\mathbb{I}\left\{E_{t}\left(s_{t}\right)\right\} V_{b},
\end{aligned}
$$

where the first inequality holds by Lemma 6, and the second inequality holds by Lemma 3 . However, by Lemma 7, the number of times the event $E_{t}\left(x_{t}\right)$ can occur is bounded by $N_{\epsilon}(\mathcal{N}+1)|\mathbb{A}| T_{\epsilon / 2}$, implying that

$$
\sum_{t=0}^{\infty} \mathbb{I}\left\{J_{M}^{\mathcal{A}_{t}}\left(x_{t}\right)<V\left(x_{t}\right)-\epsilon\right\} \leq N_{\epsilon}|\mathbb{A}|(\mathcal{N}+1) T_{\epsilon / 2},
$$

which completes the proof of the theorem, using the definition of the $\epsilon / 2$-horizon time, and the fact that $\log _{1 / \gamma} C \leq \frac{1}{1-\gamma} \ln C$, for any $C$.

\subsection{Proof of Theorem 3}

Recall the definition of the posterior number $N_{\infty}\left(x_{0}\right)$ of actually used kernels in the course of the algorithm (Definition 7). We only need to prove the analogue of Lemma 7 in this case. The rest of the proof is exactly the same as that of Theorem 1.

Thus, we need to bound the number of times that an escape event occurs. Here we consider the trees that represent the kernel sets "at infinity", namely $\Phi_{\infty}(a)=\lim _{t \rightarrow \infty} \Phi_{t}(a)$, $a \in \mathbb{A}$, instead of the $\Delta_{\epsilon}$-supported kernel set $\Phi_{\epsilon}$. As previously, the escape event can occur on internal nodes of $\Phi_{\infty}(a)$ no more than

$$
\text { (number of internal nodes of } \left.\Phi_{\infty}(a)\right) \cdot \mathcal{N} \leq N_{\infty}\left(x_{0}, a\right) \mathcal{N}
$$

times. The leaves of the tree (which are the kernels of $\Phi_{\infty}(a)$ ) can be classified into two groups: (a) "Small" leaves, with $\Delta(\phi) \leq \Delta_{\epsilon}$; and (b) "Large" leaves, with $\Delta(\phi)>\Delta_{\epsilon}$. On "small" leaves, only one occurrence of the escape event is possible, since the corresponding kernel becomes known (Definition 9) after one sample. On "large" leaves, there can not be more than $\mathcal{N}$ occurrences of the escape event-otherwise these kernels would have been split. Thus, the number of times the escape event can occur on leaves is bounded by

$$
\text { (number of leaves of } \left.\Phi_{\infty}(a)\right) \mathcal{N}=N_{\infty}\left(x_{0}, a\right) \mathcal{N} \text {. }
$$

To summarize, the number of times that the escape event can occur on all nodes, for all actions $a \in \mathbb{A}$, is bounded by $\sum_{a \in A} 2 N_{\infty}\left(x_{0}, a\right) \mathcal{N}$.

By the same arguments as in proof of Theorem 1, the above bound times the $\epsilon / 2$-horizon time $T_{\epsilon / 2}$ is the mistake bound of the algorithm.

\section{Incremental variant of the algorithm}

The ARL algorithm introduced in Sect. 4 (Algorithm 1) requires very large computational resources, as the upper value function is computed exactly each time the model is changed 
(see Algorithm 2, (13); also, see Sect. 5). In this section we propose an incremental variant of the ARL, which we call IARL (standing for Incremental ARL). This algorithm only performs back-ups of value iteration at the currently visited kernel-action pairs, instead of the exact calculation. The IARL is inspired by a similar algorithm for finite state spaces, presented in Strehl et al. (2006a). We note that intermediate versions (that perform several back-ups at each time step) can be treated similarly, but are not explicitly addressed here.

Below we present the modifications to the original algorithm. First, at each time $t$, for every action $a \in \mathbb{A}$, and for each kernel $\phi_{i}^{a} \in \Phi_{t}(a)$, we maintain an (online) estimate $\widehat{Q}_{t}(i, a)$ of $\widetilde{Q}_{t}(i, a) \triangleq \widetilde{Q}_{t}\left(\phi_{i}^{a}\right)$ (see (14)). This estimate is initialized to $\widehat{Q}_{0}(i, a) \equiv V_{\max }$. At time $t$, let

$$
U_{t}(i, a) \triangleq \tilde{r}\left(\phi_{i}^{a}\right)+\gamma\left[\min _{y \in \mathrm{UI}\left(\phi_{i}^{a}\right)} \widehat{V}_{t}(y)+\bar{\omega}\left(2 \beta \Delta\left(\phi_{i}^{a}\right)\right)\right]
$$

where

$$
\widehat{V}_{t}(x) \triangleq \max _{a \in \mathbb{A}}\left\{\sum_{i=1}^{\left|\Phi_{t}(a)\right|} \phi_{i}^{a}(x) \widehat{Q}_{t}(i, a)\right\} .
$$

Also, our incremental variant of the algorithm receives an additional parameter $\epsilon_{Q}$, which we call the upper $Q$-function accuracy parameter, to be specified in Theorem 4 below. Then, at time $t, \widehat{Q}_{t}$ is updated as follows:

$$
\widehat{Q}_{t+1}(i, a):= \begin{cases}U_{t}(i, a), & \text { if } a_{t}=a, x_{t} \in \operatorname{supp}\left(\phi_{i}^{a}\right), \text { and } U_{t}(i, a)<\widehat{Q}_{t}(i, a)-\epsilon_{Q}, \\ \widehat{Q}_{t}(i, a), & \text { otherwise. }\end{cases}
$$

That is, the update takes place only for the "visited" kernel-action pair, and only if this update changes the estimate by more than $\epsilon_{Q}$.

Each time a kernel $\phi$ is split, the upper $Q$-function estimates of the new kernels $\phi_{1}, \ldots, \phi_{K}$ are initialized to the estimate of the parent kernel $\phi$. The policy will be recomputed each time the estimates change, and it is the greedy policy with respect to $\widehat{Q}_{t}$ :

$$
\pi_{t}(x)=\operatorname{argmax}_{a \in \mathbb{A}}\left\{\sum_{i=1}^{\left|\Phi_{t}(a)\right|} \phi_{i}^{a}(x) \widehat{Q}_{t}(i, a)\right\} .
$$

To summarize, we have the following modifications to the ARL algorithm:

1. The UVF computation (15) is replaced by the update rule (39).

2. The policy computation (16) is replaced by (40).

3. The policy is recomputed each time the estimates and/or the model change.

4. The estimates of the new kernels after a split are initialized to the estimate of the parent kernel.

Now we have the following results regarding this modified algorithm.

Theorem 4 Assume that the IARL algorithm receives the input parameter $\Delta_{\epsilon}$ as in Theorem 1 as well as $\epsilon_{Q}>0$. Then, the prior mistake bound is

$$
\operatorname{PMC}\left(\epsilon+\frac{\epsilon_{Q}}{1-\gamma}\right) \leq\left(\frac{\left|\Phi_{\epsilon} \| \mathbb{A}\right|(\mathcal{N}+1)}{1-\gamma}+\frac{2\left|\Phi_{\epsilon} \| \mathbb{A}\right| V_{b}}{\epsilon_{Q}}\right) \ln \frac{2 V_{b}}{\epsilon} .
$$


In particular, choosing $\epsilon_{Q}=\epsilon(1-\gamma)$ yields

$$
\operatorname{PMC}(2 \epsilon) \leq\left(\frac{\left|\Phi_{\epsilon} \| \mathbb{A}\right|(\mathcal{N}+1)}{1-\gamma}+\frac{2\left|\Phi_{\epsilon} \| \mathbb{A}\right| V_{b}}{\epsilon(1-\gamma)}\right) \ln \frac{2 V_{b}}{\epsilon}
$$

Theorem 5 Assume that the IARL algorithm receives the input parameter $\Delta_{\epsilon}$ as in Theorem 1 as well as $\epsilon_{Q}>0$. Then, the posterior mistake bound is

$$
\operatorname{PMC}\left(\epsilon+\frac{\epsilon_{Q}}{1-\gamma}\right) \leq\left(\frac{2 N_{\infty}\left(x_{0}\right) \mathcal{N}}{1-\gamma}+\frac{2 N_{\infty}\left(x_{0}\right) V_{b}}{\epsilon_{Q}}\right) \ln \frac{2 V_{b}}{\epsilon} .
$$

In particular, choosing $\epsilon_{Q}=\epsilon(1-\gamma)$ yields

$$
\operatorname{PMC}(2 \epsilon) \leq\left(\frac{2 N_{\infty}\left(x_{0}\right) \mathcal{N}}{1-\gamma}+\frac{2 N_{\infty}\left(x_{0}\right) V_{b}}{\epsilon(1-\gamma)}\right) \ln \frac{2 V_{b}}{\epsilon} .
$$

Observe that the bounds of Theorems 4 and 5 have an additional term compared to these of Theorems 1 and 3. This term compensates for the inaccuracy that is introduced by using an estimated UVF $\widehat{V}$ for policy computation, instead of the exact UVF $\widetilde{V}$. Note that the order of magnitude of this new term is approximately $1 / \epsilon(1-\gamma)$ times that of the first term (recall the definition of $V_{b}$ in (1)).

\section{Analysis of the IARL algorithm}

The analysis of the algorithm is structured as follows. To start, we show that the estimated UVF $\widehat{V}_{t}$ indeed upper bounds the optimal value $V$. Then, we prove an analogue to Lemma 6, which states that under certain conditions on the kernel set, the optimal policy with respect to the estimated UVF is an $\epsilon$-optimal policy. Finally, we provide a bound on the number of times that the escape event can occur.

First, we prove that the estimated upper value function (38) is indeed an upper bound on the optimal value, at each time $t$.

Lemma $8 \widehat{V}_{t}(s)$ is an upper bound on the optimal value function. That is, at every time $t$, we have that

$$
\widehat{V}_{t}(x) \geq V(x), \quad \forall x \in \mathbb{X}
$$

Proof This claim is easily proved by induction on $t$. First, since $\widehat{Q}_{0}(i, a) \equiv V_{\max }$, the base case is satisfied. Now, assume that $\widehat{Q}_{t}(i, a) \geq Q(x, a)$ for all $i$ such that $x \in \operatorname{supp}\left(\phi_{i}^{a}\right)$, where $Q$ is the optimal $Q$-function. For time $t+1$, if the update of the estimated upper $Q$-function takes place for $(i, a)$, we have for all $x \in \operatorname{supp}\left(\phi_{i}^{a}\right)$, that

$$
\widehat{Q}_{t+1}(i, a)=\tilde{r}\left(\phi_{i}^{a}\right)+\gamma\left[\min _{y \in \mathrm{UI}\left(\phi_{i}^{a}\right)} \widehat{V}_{t}(y)+\bar{\omega}\left(2 \beta \Delta\left(\phi_{i}^{a}\right)\right)\right] \geq r(x, a)+\gamma V(f(x, a)) \triangleq Q(x, a),
$$

where the inequality follows by the continuity assumption and the induction assumption, similarly to the proof of Lemma 3. Finally, if the update does not take place for $(i, a)$, it holds that $\widehat{Q}_{t+1}(i, a)=\widehat{Q}_{t}(i, a) \geq Q(x, a)$ by the induction assumption. We thus have 
shown that

$$
\widehat{Q}_{t}(i, a) \geq Q(x, a), \quad \forall t, \forall(i, a): x \in \operatorname{supp}\left(\phi_{i}^{a}\right) .
$$

Hence,

$$
\widehat{V}_{t}(x) \triangleq \max _{a \in \mathbb{A}} \sum_{i=1}^{\left|\Phi_{t}(a)\right|} \phi_{i}^{a}(x) \widehat{Q}_{t}(i, a) \geq \max _{a \in \mathbb{A}} Q(x, a) \triangleq V(x), \quad \forall t, \forall x \in \mathbb{X} .
$$

To proceed, we need to obtain an analogue to Lemma 6. To that end, we introduce the following modified definitions of the known kernel-action pairs and the episode.

Definition 12 (Episode) An episode is a maximal period of time $\left[t_{0}, t_{1}\right]$, in which:

(i) All visited state-action pairs $\left(x_{t}, a_{t}\right)$ at times $t=t_{0}, \ldots, t_{1}-1$ satisfy that for each kernel $\phi_{i}^{a_{t}} \in \Phi_{t}\left(a_{t}\right)$ with $x_{t} \in \operatorname{supp}\left(\phi_{i}^{a_{t}}\right)$, it holds that

(a) $\Delta\left(\phi_{i}^{a_{t}}\right) \leq \Delta_{\epsilon}$.

(b) $\phi_{i}^{a_{t}}$ was previously sampled (that is, was previously visited).

(c) The upper $Q$-function estimate for $\phi_{i}^{a_{t}}$ satisfies:

$$
U_{t}\left(i, a_{t}\right) \geq \widehat{Q}_{t}\left(i, a_{t}\right)-\epsilon_{Q},
$$

where $U_{t}$ is defined in (37).

(ii) At time $t=t_{1}$, the algorithm encounters a pair for which the conditions in (i) are not true.

Definition 13 (Known Pairs) At any time $t$, we define the set of known kernel-action pairs:

$$
\mathcal{K}_{t} \triangleq\left\{\begin{array}{c}
\left(\phi_{i}^{a}, a\right) \in \Phi_{t}(a) \times \mathbb{A}: \Delta\left(\phi_{i}^{a}\right) \leq \Delta_{\epsilon}, \phi_{i}^{a} \text { was previously sampled, } \\
\text { and } \widehat{Q}_{t}(i, a)-U_{t}(i, a)<\epsilon_{Q}
\end{array}\right\} .
$$

The escape event is defined as previously (Definition 10). We note that the modified definition of the known pairs (and that of the escape event) depends on the size threshold parameter $\Delta_{\epsilon}$ and on the upper $Q$-function accuracy parameter $\epsilon_{Q}$. We then have the following.

Lemma 9 Let $\epsilon>0$ be given. Assume that the incremental variant receives an input $\Delta_{\epsilon}$ as in Lemma 6, and an input $\epsilon_{Q}>0$. Then,

$$
\widehat{V}_{t}(x)-J_{M}^{\mathcal{A}_{t}}(x) \leq \epsilon+\frac{\epsilon_{Q}}{1-\gamma}+\mathbb{I}\left\{E_{t}(x)\right\} V_{b}
$$

holds for all $t$ and $x \in \mathbb{X}$.

Proof Similarly to the proof of Lemma 6, at given time $t_{0}$, we consider the execution of the (non-stationary) policy $\mathcal{A}_{t_{0}}$ for $T_{\epsilon / 2}$ time steps in $M$. As previously, the escape event is expressed by the $\mathbb{I}\left\{E_{t_{0}}(x)\right\} V_{b}$ term in the bound.

Now, if during this execution we are visiting only known kernel-action pairs, for $T_{\epsilon / 2}$ time steps we stay in the same episode, and thus the algorithm's policy remains unchanged 
and it is the stationary policy $\pi_{t_{0}}$. Assume $t_{0}=0$, write $\pi$ for $\pi_{0}$ and $\widehat{V}$ for $\widehat{V}_{0}$, and recall that $\pi$ is the greedy policy with respect to $\widehat{V}$. Thus,

$$
\begin{aligned}
\widehat{V}\left(x_{0}\right) & =\sum_{\phi_{i}^{a_{0}} \in \Phi\left(a_{0}\right)} \phi_{i}^{a_{0}}\left(x_{0}\right) \widehat{Q}\left(i, a_{0}\right) \\
& \leq \sum_{\phi_{i}^{a_{0}} \in \Phi\left(a_{0}\right)} \phi_{i}^{a_{0}}\left(x_{0}\right)\left[\epsilon_{Q}+\tilde{r}\left(\phi_{i}^{a_{0}}\right)+\gamma\left(\min _{y \in \operatorname{UI}\left(\phi_{i}^{a_{0}}\right)} \widehat{V}(y)+\bar{\omega}\left(2 \beta \Delta\left(\phi_{i}^{a_{0}}\right)\right)\right)\right] \\
& =\epsilon_{Q}+\sum_{\phi_{i}^{a_{0} \in \Phi\left(a_{0}\right)}} \phi_{i}^{a_{0}}\left(x_{0}\right)\left[\tilde{r}\left(\phi_{i}^{a_{0}}\right)+\gamma\left(\min _{y \in \operatorname{UI}\left(\phi_{i}^{a_{0}}\right)} \widehat{V}(y)+\bar{\omega}\left(2 \beta \Delta\left(\phi_{i}^{a_{0}}\right)\right)\right)\right],
\end{aligned}
$$

where the inequality follows by the modified definition of the known kernel-action pairs (Definition 13). Also, by Bellman's equation,

$$
J_{M}^{\pi}\left(x_{0}\right)=r\left(x_{0}, a_{0}\right)+\gamma J_{M}^{\pi}\left(x_{1}\right) .
$$

Thus, as in the proof of Lemma 6, we have that

$$
\begin{aligned}
\widehat{V}\left(x_{0}\right)-J_{M}^{\pi}\left(x_{0}\right) & \leq \epsilon_{Q}+2 \alpha \Delta_{\epsilon}+\gamma\left(\sum_{\phi \in \Phi\left(a_{0}\right)} \phi\left(x_{0}\right)\left(\min _{y \in \mathrm{UI}(\phi)} \widehat{V}(y)+\bar{\omega}(2 \beta \Delta(\phi))\right)-J_{M}^{\pi}\left(x_{1}\right)\right) \\
& \leq \epsilon_{Q}+2 \alpha \Delta_{\epsilon}+\gamma\left(\left(\widehat{V}\left(x_{1}\right)+\bar{\omega}\left(2 \beta \Delta_{\epsilon}\right)\right)\left[\sum_{\phi \in \Phi\left(a_{0}\right)} \phi\left(x_{0}\right)\right]-J_{M}^{\pi}\left(x_{1}\right)\right) \\
& =\epsilon_{Q}+2 \alpha \Delta_{\epsilon}+\gamma \bar{\omega}\left(2 \beta \Delta_{\epsilon}\right)+\gamma\left(\widehat{V}\left(x_{1}\right)-J_{M}^{\pi}\left(x_{1}\right)\right) .
\end{aligned}
$$

Proceeding iteratively, we obtain that

$$
\begin{aligned}
\widetilde{V}\left(x_{0}\right)-J_{M}^{\pi}\left(x_{0}\right) & \leq \sum_{t=0}^{T_{\epsilon / 2}-1} \gamma^{t}\left[\epsilon_{Q}+2 \alpha \Delta_{\epsilon}+\gamma \bar{\omega}\left(2 \beta \Delta_{\epsilon}\right)\right]+\gamma^{T_{\epsilon / 2}} V_{b} \\
& \leq \frac{\epsilon_{Q}}{1-\gamma}+\frac{1}{1-\gamma}\left[2 \alpha \Delta_{\epsilon}+\gamma \bar{\omega}\left(2 \beta \Delta_{\epsilon}\right)\right]+\frac{\epsilon}{2} .
\end{aligned}
$$

Now the result follows by the precondition of the lemma on $\Delta_{\epsilon}$ (see condition (32)).

To complete the analysis, it is left to prove a bound on the number of times that the escape event can occur.

Lemma 10 The number of times that $E_{t}(x)$ can occur is bounded by

$$
\left(N_{\epsilon}|\mathbb{A}|(\mathcal{N}+1)+\frac{2 N_{\epsilon}|\mathbb{A}| V_{b}}{\epsilon_{Q}}\right) T_{\epsilon / 2} .
$$

Proof The first term in the above bound is exactly the same as that of Lemma 7. The second term addresses the modification of the definition of known kernel-action pairs in the incremental variant (Definition 13). Below we focus on each occurrence of the escape event, in which we have for the visited pair $\left(\phi_{i}^{a}, a\right)$ that $\widehat{Q}_{t}(i, a)-U_{t}(i, a)>\epsilon_{Q}$. Now, since when 
splitting is performed, we initialize the children kernels using the estimate of the parent kernel, and since we cannot decrease the estimate below its minimal possible value $V_{\min }$, it follows that the number of times that an update $\widehat{Q}_{t+1}(i, a):=U_{t}(i, a)$ can occur in a situation when $\widehat{Q}_{t}(i, a)-U_{t}(i, a)>\epsilon_{Q}$, is bounded by $V_{b} / \epsilon_{Q}$. We wish to bound the number of such updates for all possible kernel-action pairs in the course of learning. Hence, the above bound should be multiplied by $2 N_{\epsilon}|\mathbb{A}| T_{\epsilon / 2}$, using similar arguments to those of the proof of Lemma 7.

Finally, we prove the prior and the posterior mistake bounds for the incremental variant.

Proof of Theorem 4 For each time $t$, we consider the execution of policy $\mathcal{A}_{t}$ for $T_{\epsilon / 2}$ timesteps in $M$, with the initial state in each such execution $x_{t}$. We then have that

$$
\begin{aligned}
J_{M}^{\mathcal{A}_{t}}\left(x_{t}\right) & \geq \widehat{V}_{t}\left(x_{t}\right)-\epsilon-\frac{\epsilon_{Q}}{1-\gamma}-\mathbb{I}\left\{E_{t}\left(x_{t}\right)\right\} V_{b} \\
& \geq V\left(x_{t}\right)-\epsilon-\frac{\epsilon_{Q}}{1-\gamma}-\mathbb{I}\left\{E_{t}\left(x_{t}\right)\right\} V_{b},
\end{aligned}
$$

where the first inequality holds by Lemma 9, and the second inequality holds by Lemma 8 . However, by Lemma 10 , the number of times the event $E_{t}\left(x_{t}\right)$ can occur is bounded by $\left(N_{\epsilon}(\mathcal{N}+1)|\mathbb{A}|+\frac{2 N_{\epsilon}|\mathbb{A}| V_{b}}{\epsilon_{Q}}\right) T_{\epsilon / 2}$, implying that

$$
\sum_{t=0}^{\infty} \mathbb{I}\left\{J_{M}^{\mathcal{A}_{t}}\left(x_{t}\right)<V\left(x_{t}\right)-\epsilon-\frac{\epsilon_{Q}}{1-\gamma}\right\} \leq\left(N_{\epsilon}|\mathbb{A}|(\mathcal{N}+1)+\frac{2 N_{\epsilon}|\mathbb{A}| V_{b}}{\epsilon(1-\gamma)}\right) T_{\epsilon / 2},
$$

which completes the proof of the theorem, using the definition of the $\epsilon / 2$-horizon time, and the fact that $\log _{1 / \gamma} C \leq \frac{1}{1-\gamma} \ln C$, for any $C$.

Proof of Theorem 5 The proof of the posterior mistake bound of the incremental variant is similar to that of Theorem 3, with the modified definition of known cell-action pairs, as it was done above for the prior bound.

\section{Practical implementation of the ARL}

In this section we discuss the applicability of the ARL algorithm and its assumptions to a simple deterministic control problem, namely to the problem of inverted pendulum. In addition, we discuss some improvements and extensions that can increase the practical efficiency of the algorithm.

\subsection{The inverted pendulum problem}

An inverted pendulum has its mass above its pivot point. The inverted pendulum is inherently unstable, and must be actively balanced in order to remain upright. Below we show that the assumptions in this paper hold for this problem. We consider the simplest version of this problem, in which the control is performed by applying a torque at a fixed pivot point, as shown in Fig. 3. Moreover, we restrict the action set to $\mathbb{A}=\{-1,1\}$, which corresponds to application of negative and positive torque, respectively. 
Fig. 3 A schematic drawing of the inverted pendulum

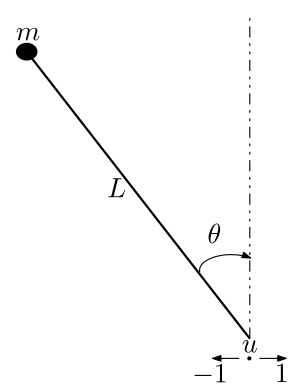

The dynamics of this system can be written as follows:

$$
\ddot{\theta}(t)=\frac{m g L}{J} \sin (\theta(t))+\frac{u}{J},
$$

where $\ddot{\theta}$ is the angular acceleration, $g$ is the gravitational acceleration, $u \in \mathbb{A}$ is the control variable (that is, the torque applied at the pivot point), and $J$ is the pendulum moment of inertia.

The discrete time Euler's approximation to this equation is

$$
\begin{aligned}
& x_{n+1}^{a}=x_{n}^{a}+h x_{n}^{b} \triangleq f^{a}\left(x_{n}, u\right), \\
& x_{n+1}^{b}=x_{n}^{b}+h\left(\frac{m g L}{J} \sin \left(x_{n}^{a}\right)+\frac{u}{J}\right) \triangleq f^{b}\left(x_{n}, u\right),
\end{aligned}
$$

where the state is the pair $x \triangleq\left(x^{a}, x^{b}\right)=(\theta, \dot{\theta})$ with $^{5}|\theta| \leq \pi$ and $|\dot{\theta}| \leq \dot{\theta}_{\text {max }}$, and $h>0$ is the step size parameter. It is easily verified that the transition function $f: \mathbb{X} \times \mathbb{A} \rightarrow \mathbb{X}$ satisfies Assumption 2. In particular, assuming that the infinity norm distance is used, the continuity coefficient $\beta$ can be taken as

$$
\beta=\max \left\{1+h, 1+h \frac{m g L}{J}\right\} .
$$

We consider below the following situation. Suppose that the dynamics of the system are unknown, while the reward function is to be chosen by the planner in order to accommodate the prescribed task. Specifically, in this problem we are interested in balancing the pendulum in the upward position. Therefore, the reward function ideally should be

$$
r(x, u)= \begin{cases}1, & x=(0,0) \\ 0, & \text { otherwise }\end{cases}
$$

However, this function is clearly useless since we cannot expect any algorithm to "hit" the origin exactly, implying that the estimated reward will always be zero. Hence, a smoothed version of this reward function should be chosen. For example, using the infinity norm dis-

\footnotetext{
${ }^{5}$ We note that we can always take the interval $\left[-\dot{\theta}_{\max }, \dot{\theta}_{\max }\right]$ large enough to ensure that the angular velocity falls in this interval during the learning process.
} 
Fig. 4 An approximation of the optimal policy and two particular optimal trajectories for the inverted pendulum problem, using exact computation on a dense uniform grid. In the darker regions the optimal action is -1 , while in the lighter regions the optimal action is 1

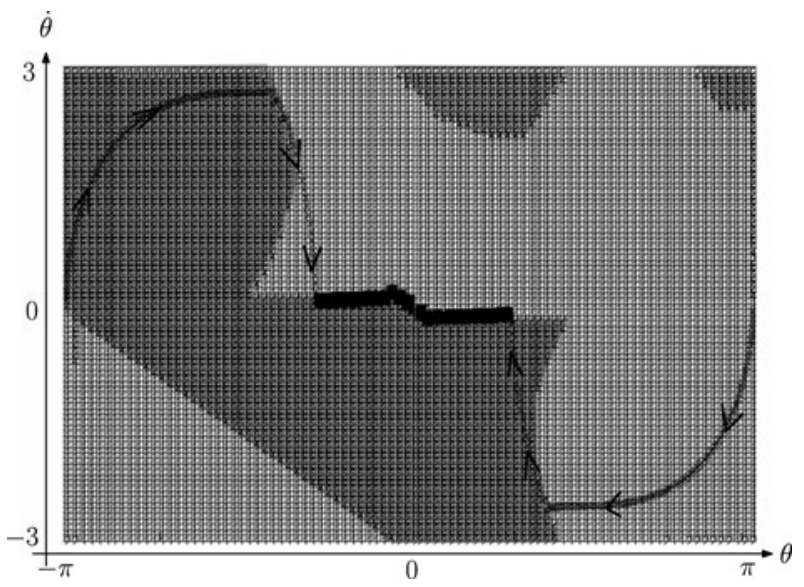

Fig. 5 The common grid produced by the ARL in the long term, after convergence to the marked trajectory

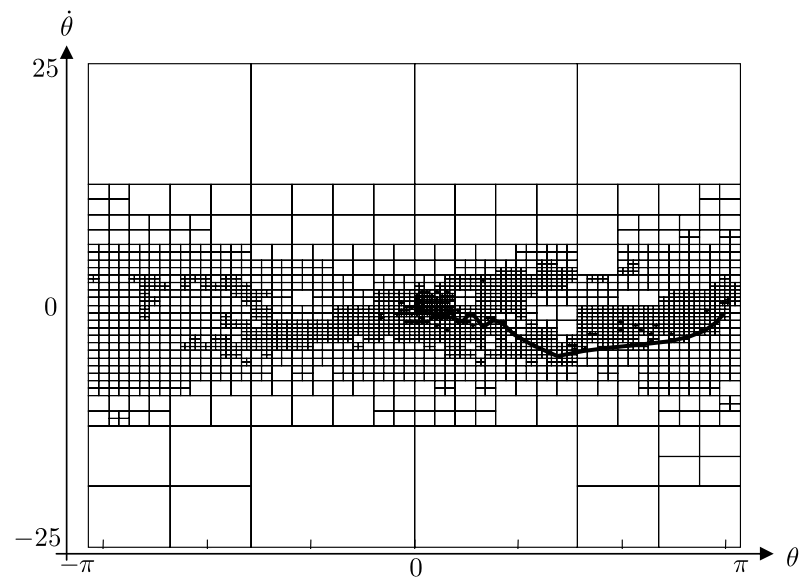

tance to the origin, we can set:

$$
r(x, u)= \begin{cases}1-\max \left\{\left|\frac{x^{a}}{K_{a}}\right|,\left|\frac{x^{b}}{K_{b}}\right|\right\}, & \left|x^{a}\right| \leq K_{a},\left|x^{b}\right| \leq K_{b} \\ 0, & \text { otherwise }\end{cases}
$$

where $K_{a} \leq \pi$ and $K_{b} \leq \dot{\theta}_{\max }$. In this case, we have that the reward continuity coefficient $\alpha=\max \left\{1 / K_{a}, 1 / K_{b}\right\}$.

We applied the ARL algorithm with B-splines of degree 0 (that is, strict state aggregation) to this problem. The goal of the simulation was to examine the density of the adaptive grid produced by the algorithm in the long term. We performed repeated episodial runs, with each episode starting at the same initial state $(\theta, \dot{\theta})=(\pi, 0)$. Each time the algorithm reached the vicinity of the target state $(0,0)$, it was restarted from the initial position. Some illustrative results can be seen in Figs. 4 and 5. Figure 4 shows an optimal policy and two particular optimal trajectories obtained by exact computation using a dense uniform grid, with cell size of 0.07 . Figure 5 shows the (common) $\operatorname{grid}^{6}$ produced by the ARL in the long term.

\footnotetext{
${ }^{6}$ See the Appendix for the definition of the common grid in the case of strict state aggregation.
} 
(Note the scale change in the $\dot{\theta}$ axis.) The initial kernel set $\Phi_{0}$ consisted of a single kernel covering the whole state space (with support diameter of 50). A discount factor of $\gamma=0.99$ was used, the splitting scheme parameters were $K=4$ and $\lambda=0.5$, and the algorithm was executed with the threshold parameters $\mathcal{N}=10$ and $\Delta_{\epsilon}=0.25$. The differential equation (41) was discretized using $h=0.05$. The simulation was stopped when there was no change in the grid from run to run. The total number of cells after convergence was $N_{\infty}\left(x_{0}\right)=5280$; compare to $\left|\Phi_{\epsilon}\right||\mathbb{A}| \approx 480000$, which can be estimated using Lemma 4. We note that the fine resolution in the left part of the state space is due to the second optimal trajectory (cf. Fig. 4), which was occasionally traversed by the algorithm during the learning process. This example supports the conjecture that the actual number of kernels in the long term should be much less than $\left|\Phi_{\epsilon} \| \mathbb{A}\right|$. Therefore, the bound of Theorem 3 is indeed considerably tighter than that of Theorem 1.

\subsection{Ideas for enhancing ARL}

Our goal in this paper was to develop the simplest possible adaptive-resolution algorithm for which formal guarantees can be obtained, in order to emphasize the related essential ideas. Therefore, we used a very simple splitting criterion. For practical implementations, the proposed algorithm may be modified and extended to make its actual performance (rather than the performance bound) more efficient. We list below few ideas for such extensions, leaving the analysis of their effect to future work.

Splitting and merging rules More elaborate splitting rules and possible merging schemes should be considered, as for example in Munos and Moore (2002) and Bonarini et al. (2005). For instance, one may consider to merge kernels based on recency of the visits or homogeneity of the values. Regarding the splitting criterion, it maybe useful to employ a variable count threshold $\mathcal{N}_{t}$. In particular, it seems reasonable to start with a relatively large threshold and gradually decrease it. This will eliminate numerous splits at an early stage of the algorithm and will allow it to explore the state space. As time progresses, the threshold should be decreased in order to boost the convergence to the optimal trajectory.

Multiple samples In principle, our algorithm uses a single sample to estimate the model. However, as was already mentioned in Sect. 4.2, more efficient empirical models may be used. For example, one may replace a sample with a "better" new sample (see Sect. 4.2 for a possible meaning of "better"). Moreover, all the samples can be saved and used in order to define the model. This is obviously necessary when considering stochastic domains, but can be also useful in deterministic ones. The only requirement is that the obtained model will be "optimistic" in the sense that the rewards are upper bounded and the transitions are in the corresponding uncertainty set.

Non-uniform modulus of continuity Our algorithm uses uniform continuity coefficients $\alpha$ and $\beta$. In practice, it may be possible to obtain tighter non-uniform coefficients $\alpha(x)$ and $\beta(x)$. For example, consider a continuous approximation of the reward function for the inverted pendulum problem given in (42). This function is zero in most of the regions of the state space. If we know that in advance (which is certainly the case when the planner sets the reward function), we can just use a coefficient $\alpha=0$ for these regions. As a result, the convergence of the algorithm may be boosted since there is no need to "explore" these regions, at least as far as the reward function is concerned. Moreover, if the agent knows the exact reward function, he can use the actual upper bound on the reward in each kernel (instead of the upper bound that is based on the continuity coefficient $\alpha$ ). 


\section{Conclusion}

We presented a learning algorithm which combines a model-based approach and kernelbased value function approximation, in order to solve the online, continuous state space reinforcement learning problem in deterministic domains, under continuity assumption of model parameters. We note that we focused on deterministic domains for simplicity, but we believe that the proposed approach and results can be extended to stochastic systems by adding stochastic confidence bounds.

Our analysis was meant to show feasibility in the sense of sample efficiency. To our best knowledge, this is the first online performance bound result for kernel-based RL algorithm in continuous state space. In addition, an incremental variant was presented and analyzed. This variant is a more practical version of the original algorithm, with reduced computational complexity at each stage.

Two types of mistake bounds were established: prior and posterior. The prior bound is similar to the bound for the finest resolution model (up to factor $\mathcal{N}+1$ ), and is not necessarily obtained by a naïve approach, where the finest resolution model is treated as a finite-state MDP, and an efficient exploration technique is used on this MDP (as discussed in Sect. 6). The posterior bound is expressed in terms of the actual kernel set discovered in the course of learning. As far as we know, this is the first time that a bound of this type was established for RL in continuous state spaces. As the simulation results presented in Sect. 10.1 suggest, this bound should be much tighter in practice than the prior bound.

It is worthwhile to mention that the prior bound is new even in the non-adaptive case, when we start the ARL with the finest resolution model from the outset (cf. Theorem 2). Thus, we consider the prior bound a novel contribution of this paper to the theory of RL.

We note that, ideally, one would want the bound to be in terms of "the best" or "natural" kernel set for the domain. This can be defined, for example, as the minimal kernel set needed in order to obtain an $\epsilon$-optimal policy. However, how to discover such an "optimal" kernel set remains an open and difficult question (even in an offline setting) which requires new tools for its analysis.

Finally, we list below some ideas for future work.

- Evaluation of the algorithm and possible variants using extensive simulations would be interesting from the practical point of view.

- The effect of the enhancements proposed in Sect. 10.2 on the mistake bounds should be analyzed.

- The extension of similar ideas to the stochastic domains seems possible, under a different continuity assumption (namely, under continuity of transition density as in Chow and Tsitsiklis 1991). Preliminary results for the special case of adaptive aggregation can be found in Bernstein (2007). A possible future direction here is to formulate an algorithm that will work for both the stochastic and deterministic cases, under a unified continuity assumption, similarly to the approach taken by Nouri and Littman (2008).

- The model used in this paper assumes a finite action space. We note that the proposed algorithm can be applied also to continuous action space by using some fixed (predefined) discretization of the action space. More efficient algorithm may possibly be obtained by using adaptive aggregation of the action space, similar to that of the state space.

- Other reward criteria should be considered-average reward (with associated loss bounds), and shortest path problems (total reward). In particular, the shortest path formulation is often associated with such deterministic problems, as navigation in a maze.

Acknowledgements We would like to thank the reviewers and the action editor for helpful comments and suggestions. 


\section{Appendix}

Below we discuss two important special cases of the general kernel-based framework, where the UVF computation procedure proposed in Sect. 5 can be carried out in an efficient way.

Constant kernels—state aggregation

Consider the important special case where constant kernels are used. Namely, for all $a \in \mathbb{A}$ and $\phi_{i}^{a} \in \Phi_{t}(a)$ we have that

$$
\phi_{i}^{a}(x)=\mathbb{I}\left\{x \in \operatorname{supp}\left(\phi_{i}^{a}\right)\right\}
$$

and only one kernel is active for each $x$. These kernels correspond to B-splines of the degree 0, in the terminology of Unser (1999). Observe that our general framework in this case reduces to (adaptive) grid-based representation of the state space, since each "kernel" aggregates a part of the state space, and there is no overlap in the supports of different kernels in the same action kernel set. A splitting scheme in this case is simply dividing a cell (that is, the support of the corresponding kernel) into disjoint subcells which cover the original cell.

Also, the computational procedure described in Sect. 5 reduces to that of the Adaptive Aggregation Algorithm (AAA) (Bernstein and Shimkin 2008). ${ }^{7}$ Indeed, the UVF $\widetilde{V}_{t}(x)$ is a piecewise constant function and the minimization in (20) reduces to a minimum of a finite number of values. In particular, let

$$
\mathbb{S}_{t}(a)=\left\{s=\operatorname{supp}(\phi): \phi \in \Phi_{t}(a)\right\}
$$

be the grid that is used by the algorithm at time $t$ for action $a$. We denote by $\mathbb{S}_{t}$ the coarsest grid which is a refinement of all $\mathbb{S}_{t}(a)$ at time $t$. That is

$$
\mathbb{S}_{t} \triangleq \bigwedge_{a \in \mathbb{A}} \mathbb{S}_{t}(a)
$$

where the intersection operator is defined as:

$$
A \wedge B \triangleq\left\{s_{A} \cap s_{B}: s_{A} \in A, s_{B} \in B\right\} \backslash\{\varnothing\} .
$$

We call $\mathbb{S}_{t}$ the common grid at time $t$. Using this notation, we can rewrite (20) for the case of constant kernels as follows:

$$
\widetilde{T}(s, a)=\min _{s^{\prime} \in \mathrm{UI}_{f}(s, a)} \max _{a^{\prime} \in \mathbb{A}}\left\{\tilde{r}\left(s^{\prime}, a^{\prime}\right)+\gamma\left[\widetilde{T}\left(s^{\prime}, a^{\prime}\right)+\bar{\omega}\left(s^{\prime}, a^{\prime}\right)\right]\right\}, \quad(s, a) \in \mathbb{S}_{t}(a) \times \mathbb{A} .
$$

Here, $\mathrm{UI}_{f}: \mathbb{S}_{t}(a) \times \mathbb{A} \rightarrow \mathbb{S}_{t}, \tilde{r}: \mathbb{S}_{t}(a) \times \mathbb{A} \rightarrow \mathbb{R}$, and $\bar{\omega}: \mathbb{S}_{t}(a) \times \mathbb{A} \rightarrow \mathbb{R}$ are corresponding uncertainty parameters (obtained similarly to those of the general kernel-based framework) which are naturally extended to $\mathbb{S}_{t} \times \mathbb{A}$. Now, observe that since the minimum and maximum in (43) are over finite sets, the related computational procedure can be carried out efficiently.

\footnotetext{
${ }^{7}$ We note however that the proposed algorithm differs from the AAA algorithm as explained in the introduction section. 


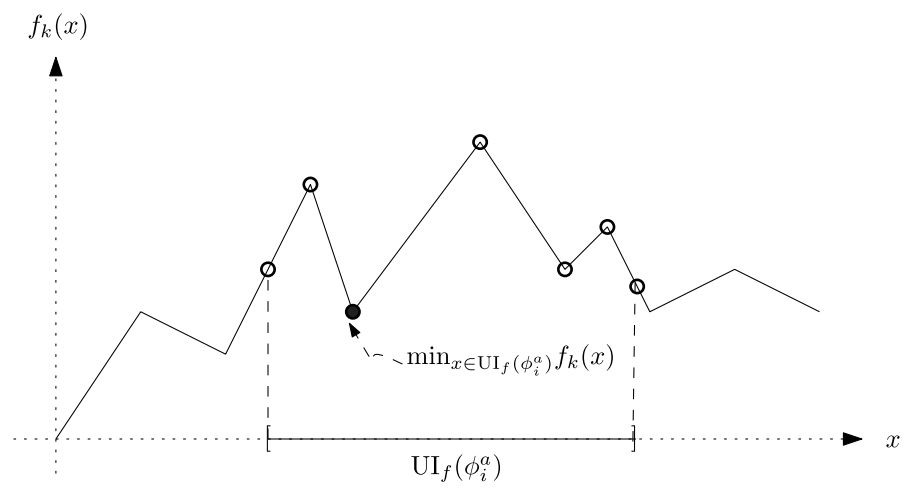

Fig. 6 Minimization problem in the case of triangular kernels. Empty circles denote the set $G(i, a)$ of "knot" and boundary points. The minimum of $f_{k}$ is attained at the point denoted by the filled circle

Triangular kernels_-piecewise-linear approximation

Here we consider the special case where triangular kernels are used. An example of such kernel is shown in Fig. 1 for one-dimensional case. These kernels correspond to B-splines of the degree 1 . The construction of higher-dimensional triangular kernels can be done for example using tensor-product basis functions as in Unser (1999).

Uniform splitting schemes in this case should be considered. One possibility is to use a standard B-spline "pyramids" as discussed in Sect. 3.2 (see Fig. 1). We note that other splitting schemes, which satisfy Definition 3 are also possible.

Regarding the policy computation procedure, observe that in this case the UVF $\widetilde{V}_{t}(x)$ is a piecewise linear function (as it is a finite maximum of piecewise linear functions by its definition). Thus, the minimum in (20) is achieved at the non-differentiable points of its argument (that is, at the "knot" points). This fact may considerably simplify the computation of (20), since the cardinality of the set of such knot points is much smaller than cardinality of $\mathrm{UI}_{f}$.

For instance, consider the one-dimensional case, recall the general computational procedure (21), and set

$$
f_{k}(x) \triangleq \max _{a^{\prime} \in \mathbb{A}}\left\{\sum_{j=1}^{\left|\Phi_{t}\left(a^{\prime}\right)\right|} \phi_{j}^{a^{\prime}}(x)\left(\tilde{r}\left(\phi_{j}^{a^{\prime}}\right)+\gamma\left[\widetilde{T}^{(k-1)}\left(j, a^{\prime}\right)+\bar{\omega}\left(j, a^{\prime}\right)\right]\right)\right\} .
$$

Note that $f_{k}(x)$ is a piecewise-linear function since it is a finite maximum of linear combination of triangular kernels. Now, the update required in (21) is

$$
\widetilde{T}^{(k)}(i, a)=\min _{x \in \mathrm{UI}_{f}\left(\phi_{i}^{a}\right)} f_{k}(x),
$$

which is a minimum of the piecewise-linear function $f_{k}(x)$ over the convex set $\mathrm{UI}_{f}\left(\phi_{i}^{a}\right)$. Let

$$
G(i, a)=\left\{x \in \mathrm{UI}_{f}\left(\phi_{i}^{a}\right): f_{k}(x) \text { is non-differentiable at } x\right\} \cup \partial \mathrm{UI}_{f}\left(\phi_{i}^{a}\right),
$$

where $\partial \mathrm{UI}_{f}\left(\phi_{i}^{a}\right)$ is the boundary of $\mathrm{UI}_{f}\left(\phi_{i}^{a}\right)$. However, $|G(i, a)|$ is finite since $f_{k}(x)$ is piecewise-linear. Thus, the update

$$
\widetilde{T}^{(k)}(i, a)=\min _{x \in \mathrm{UI}_{f}\left(\phi_{i}^{a}\right)} f_{k}(x)=\min _{x \in G(i, a)} f_{k}(x)
$$

can be carried out efficiently, as illustrated by Fig. 6 . 


\section{References}

Albus, J. S. (1975). A new approach to manipulator control: the cerebellar model articulation controller (CMAC). Journal of Dynamic Systems, Measurement and Control, 97, 220-227.

Antos, A., Szepesvári, C., \& Munos, R. (2008). Learning near-optimal policies with Bellman-residual minimization based fitted policy iteration and a single sample path. Machine Learning, 71(1), 89-129.

Auer, P., \& Ortner, R. (2006). Logarithmic online regret bounds for undiscounted reinforcement learning. In Proceedings of neural information processing systems conference (NIPS).

Bernstein, A. (2007). Adaptive state aggregation for reinforcement learning. Master's thesis, TechnionIsrael Institute of Technology. URL: http://tx.technion.ac.il/ andreyb/MSc_Thesis_final.pdf.

Bernstein, A., \& Shimkin, N. (2008). Adaptive aggregation for reinforcement learning with efficient exploration: deterministic domains. In Proceedings of the 21st annual conference on learning theory (COLT 2008).

Bertsekas, D. P. (2007). Dynamic programming and optimal control (3rd ed., vol. 2). Belmont: Athena Scientific.

Bonarini, A., Lazaric, A., \& Restelli, M. (2005). LEAP: learning entities adaptive partitioning. In Proceedings of neural information processing systems conference (NIPS 2005), workshop on reinforcement learning benchmarks and bake-offs II, Whistler, Canada (pp. 41-47).

Boutilier, C., Dean, T., \& Hanks, S. (1999). Decision-theoretic planning: structural assumptions and computational leverage. Journal of Artificial Intelligence Research, 11, 1-94.

Brafman, R. I., \& Tennenholtz, M. (2002). R-MAX - a general polynomial time algorithm for near-optimal reinforcement learning. Journal of Machine Learning Research, 3, 213-231.

Chapman, H. (2007). Global confidence bound algorithms for the exploration-exploitation tradeoff in reinforcement learning. Master's thesis, Technion-Israel Institute of Technology.

Chow, C.-S., \& Tsitsiklis, J. N. (1991). An optimal one-way multigrid algorithm for discrete-time stochastic control. IEEE Transactions on Automatic Control, 36(8), 898-914.

Doya, K. (2000). Reinforcement learning in continuous time and space. Neural Computation, 12, $219-245$.

Jong, N., \& Stone, P. (2006). Kernel-based models for reinforcement learning in continuous state spaces. In 23th international conference on machine learning (ICML 2006), workshop on kernel machines and reinforcement learning.

Kakade, S. M. (2003). On the sample complexity of reinforcement learning. PhD thesis, Gatsby Computational Neuroscience Unit, University College London, UK.

Kearns, M., \& Singh, S. P. (2002). Near-optimal reinforcement learning in polynomial time. Machine Learning, 49, 209-232.

Konda, V. R., \& Tsitsiklis, J. N. (2003). On actor-critic algorithms. SIAM Journal on Control and Optimization, 42(4), 1143-1166.

Loth, M., Davy, M., Coulom, R., \& Preux, P. (2006) Equi-gradient temporal difference learning. In 23th international conference on machine learning (ICML 2006), workshop on kernel machines and reinforcement learning.

Moore, A. W., \& Atkeson, C. G. (1995). The parti-game algorithm for variable resolution reinforcement learning in multidimensional state-spaces. Machine Learning, 21, 199-233.

Munos, R., \& Moore, A. W. (2002). Variable resolution discretization in optimal control. Machine Learning, 49, 291-323.

Munos, R., \& Szepesvári, C. (2008). Finite-time bounds for fitted value iteration. Journal of Machine Learning Research, 9, 815-857.

Nouri, A., \& Littman, M. L. (2008). Multi-resolution exploration in continuous spaces. In Advances in neural information processing systems (NIPS) 21 (pp. 1209-1216).

Ormoneit, D., \& Sen, S. (2002). Kernel-based reinforcement learning. Machine Learning, 49, 161-178.

Powell, W. B. (2007). Approximate dynamic programming for operations research: solving the curses of dimensionality. New York: Wiley.

Puterman, M. L. (1994). Markov decision processes: discrete stochastic dynamic programming. New York: Wiley.

Singh, S. P., Jaakkola, T., \& Jordan, M. I. (1995). Reinforcement learning with soft state aggregation. In Advances in neural information processing systems (NIPS) 7 (pp. 361-368).

Strehl, A. L., \& Littman, M. L. (2005). A theoretical analysis of model-based interval estimation. In Proceedings of the 22nd international conference on machine learning (pp. 857-864).

Strehl, A. L., Li, L., \& Littman, M. L. (2006a). Incremental model-based learners with formal learning-time guarantees. In Proceedings of the 22nd international conference on uncertainty in artificial intelligence (pp. 485-493).

Strehl, A. L., Wiewiora, E., Langford, J., \& Littman, M. L. (2006b). PAC model-free reinforcement learning. In Proceedings of the 23nd international conference on machine learning (pp. 881-888). 
Sutton, R. S. (1996). Generalization in reinforcement learning: successful examples using sparse coarse coding. In Advances in neural information processing systems 8 (NIPS) (pp. 1038-1044).

Tewari, A., \& Bartlett, P. L. (2007). Optimistic linear programming gives logarithmic regret for irreducible MDPs. In Proceedings of neural information processing systems conference (NIPS).

Unser, M. (1999). Splines: A perfect fit for signal and image processing. IEEE Signal Processing Magazine, 16, 22-38.

Whitt, W. (1978). Approximations of dynamic programs, I. Mathematics of Operations Research, 3(3), 231243. 